\title{
COMPUTATIONAL ANALYSIS OF
}

\section{GRAVITATIONAL EFFECTS IN LOW-DENSITY GAS JETS}

\author{
Rajani P. Satti and Ajay K. Agrawal ${ }^{1}$ \\ School of Aerospace and Mechanical Engineering \\ University of Oklahoma, Norman, OK-73019
}

\begin{abstract}
This study deals with the computational analysis of buoyancy-induced instability in the nearfield of an isothermal helium jet injected into quiescent ambient air environment. Laminar, axisymmetric, unsteady flow conditions were considered for the analysis. The transport equations of helium mass fraction coupled with the conservation equations of mixture mass and momentum were solved using a staggered grid finite volume method. The jet Richardson numbers of 1.5 and 0.018 were considered to encompass both buoyant and inertial jet flow regimes. Buoyancy effects were isolated by initiating computations in Earth gravity and subsequently, reducing gravity to simulate the microgravity conditions. Computed results concur with experimental observations that the periodic flow oscillations observed in Earth gravity subside in microgravity.
\end{abstract}

Keywords: Buoyancy, Inertial, Flow computations, Flow Instability

\footnotetext{
${ }^{1}$ Corresponding Author

Senior Member of AIAA, Lloyd G. and Joyce Austin Presidential Professor and Associate Professor 865 Asp Avenue, Room 212, School of Aerospace and Mechanical Engineering University of Oklahoma, Norman, OK 73019

Phone: (405) 325-1754, Fax: (405)325-1088, email: aagrawal@ou.edu
} 


\section{INTRODUCTION}

Low-density gas jets are characterized by the injection of a lighter fluid into a dense ambient environment. The resulting flow structure is susceptible to buoyancy arising because of the difference in densities of jet and surrounding fluids. In general, jets are considered buoyant for Richardson number, $\mathrm{Ri}=\operatorname{gd}\left(\rho_{\infty}-\rho_{\mathrm{j}}\right) / \rho_{\mathrm{j}} \mathrm{U}_{\mathrm{j}}^{2}>1.0$ and inertial for $\mathrm{Ri}<<1.0$, where $\mathrm{g}$ is the gravitational acceleration, $d$ is the injector tube inside diameter, $\rho_{\infty}$ and $\rho_{j}$ are, respectively, the ambient and jet fluid densities, and $U_{j}$ is the average jet exit velocity. The present study examines low-density gas jets in both buoyant and inertial flow regimes, with the goal of delineating gravitational effects on the flow structure in the near-field.

Several studies ${ }^{1-6}$ have focused on the far-field behavior of low-density jets, which is influenced by the instabilities arising in the near-field. Low-density gas jets are known to exhibit self-excited periodic oscillations in both buoyant and the inertial flow regimes. In the buoyant flow regime, Subbarao and Cantwell ${ }^{7}$ conducted experiments with helium jets injected into a coflow of air for Ri varying from 0.5 to 6.0. The frequency (f) of the periodic oscillations expressed by the Strouhal number, $\mathrm{St}=\mathrm{fd} / \mathrm{Uj}$ correlated with Richardson number for $\mathrm{Ri}>1.0$ indicating buoyancy dependent instability. Hamins et al. ${ }^{8}$ observed periodic oscillations in a helium jet injected into air, and reported that a minimum jet exit velocity was required to initiate the oscillations. Similar experiments were conducted by Cetegen and co-workers ${ }^{9-11}$ for axisymmetric and planar plumes, whereby buoyancy induced toroidal vortical structures contaminating the primary jet flow were observed. Diagnostics involved flow measurements using laser Doppler velocimetry and particle image velocimetry. Pasumarthi and Agrawal $^{12}$ conducted experiments to characterize jet flow in terms of concentration measurements across 
the whole field of a helium jet injected into quiescent air. Experiments by Yep et al. ${ }^{13}$ demonstrated that the self-excited flow oscillations in Earth gravity were absent in the microgravity environment of the $2.2 \mathrm{~s}$ drop tower.

Self-excited oscillations have also been reported in low-density inertial jets at $\mathrm{Ri}<<1.0^{14-17}$. In these studies, buoyancy effects were considered negligible because of the small Richardson number. Although this assumption is justified in the jet core, buoyancy may be significant in the jet shear layer where density and velocity gradients are large. Recently, Pasumarthi ${ }^{18}$ conducted experiments at $\mathrm{Ri}<1.0$ using helium jet injected into quiescent air in the microgravity environment of the $2.2 \mathrm{~s}$ drop tower. Concentration measurements revealed global oscillations in the near-field in Earth gravity. In microgravity, the oscillation amplitude decreased significantly for $\mathrm{Ri}<0.01$ and no oscillations were observed for $\mathrm{R} i>0.1$. These experiments provided direct physical evidence for the first time that the flow oscillations in inertial low-density gas jets are buoyancy-induced, a well known phenomenon in buoyant jets.

Past experiments have characterized buoyancy effects using either concentration or velocity measurements. However, simultaneous visualization of velocity and concentration fields is desired to fully understand the dynamics of the oscillating flow structure. Experimental difficulties in obtaining simultaneous vector-scalar measurements, especially in the low-gravity environment, have prompted studies of gas jets using computational fluid dynamic (CFD) analysis. Mell et al. ${ }^{19}$ performed numerical simulations of a helium jet injected into air and found that the computed oscillation frequency matched with experiments of Hamins et $\mathrm{ll}^{8}$. Recently, Soteriou et al. ${ }^{20}$ investigated the near-field dynamics of planar buoyant plumes incorporating a Lagrangian transport element method. Computations qualitatively captured the plume instantaneous behavior observed experimentally. The mechanism of vorticity generation 
and relative roles played by buoyancy and viscous forces in explaining the plume instability were discussed. However, a description of the flow field and its interaction with the scalar structure is desired to explain the instability in low-density gas jets. On a related note, several numerical studies have been conducted to understand the dynamics of flickering jet diffusion flames ${ }^{21,22}$. Thus, the objective of the present study is to delineate the effects of buoyancy in low-density gas jets. The CFD analysis is utilized to concurrently visualize the velocity and concentration fields. Both buoyant and inertial jets are examined in Earth gravity, microgravity, and during change from Earth to microgravity.

\section{NUMERICAL FORMULATION}

\subsection{Governing Equations}

Consistent with experimental studies ${ }^{12,13}$, the numerical formulation is based on unsteady, laminar, and axisymmetric flow behavior. The following additional approximations were made to simplify the analysis: (i) the flow is incompressible, (ii) temperature and pressure variations are negligible and (iii) the flow is a binary fluid system with air treated as a single species. Accordingly, the governing equations are expressed as follows:

$$
\begin{aligned}
& \frac{\partial \rho}{\partial t}+\frac{1}{r} \frac{\partial}{\partial r}(\rho v r)+\frac{\partial}{\partial z}(\rho w)=0 \\
& \frac{\partial(\rho r \phi)}{\partial t}+\frac{\partial}{\partial r}(\rho v r \phi)+\frac{\partial}{\partial z}(\rho r w \phi)=\frac{\partial}{\partial r}\left(\Gamma^{\phi} r \frac{\partial \phi}{\partial r}\right)+\frac{\partial}{\partial z}\left(\Gamma^{\phi} r \frac{\partial \phi}{\partial z}\right)+S^{\phi}
\end{aligned}
$$

Equation (1) represents the continuity equation and Equation (2) is the generalized transport equation, which represents the momentum, or species conservation equation depending on the 
variable $\phi . \Gamma^{\phi}$ and $S^{\phi}$ are, respectively, the transport coefficient and source term of the variable $\phi$, as listed in Table 1 .

Table 1. Transport coefficients and source terms in Equation 2

\begin{tabular}{|c|c|c|c|}
\hline Variable & $\phi$ & $\Gamma^{\phi}$ & $S^{\phi}$ \\
\hline $\begin{array}{c}\text { Axial } \\
\text { Velocity }\end{array}$ & $\mathrm{w}$ & $\mu$ & $-r \frac{\partial p}{\partial z}+r\left(\rho_{\infty}-\rho\right) g+\frac{\partial}{\partial z}\left(r \mu \frac{\partial w}{\partial z}\right)+\frac{\partial}{\partial r}\left(r \mu \frac{\partial v}{\partial z}\right) \frac{2}{3}\left[\frac{\partial}{\partial z}\left(r \mu \frac{\partial v}{\partial r}\right)+\frac{\partial}{\partial z}\left(r \mu \frac{\partial v}{\partial z}\right)\right]$ \\
\hline $\begin{array}{c}\text { Radial } \\
\text { Velocity }\end{array}$ & $\mathrm{v}$ & $\mu$ & $-r \frac{\partial p}{\partial r}-\frac{2}{3} \mu \frac{v}{r}+\frac{\partial}{\partial z}\left(r \mu \frac{\partial w}{\partial r}\right)+\frac{\partial}{\partial r}\left(r \mu \frac{\partial v}{\partial r}\right)-\frac{2}{3}\left[\frac{\partial}{\partial r}\left(r \mu \frac{\partial v}{\partial r}\right)+\frac{\partial}{\partial r}\left(r \mu \frac{\partial w}{\partial z}\right)\right]$ \\
\hline $\begin{array}{c}\text { Helium } \\
\text { mass } \\
\text { fraction }\end{array}$ & $\mathrm{f}$ & $\rho \mathrm{D}_{\mathrm{b}}$ & 0 \\
\hline
\end{tabular}

Density was computed from the equation of state for incompressible ideal gas. The dynamic viscosity was calculated using Wilke's mixture averaged formula ${ }^{23}$. The binary diffusion coefficient of helium into air, $D_{b}$ was specified as a constant. ${ }^{24}$ In microgravity, the gravitational acceleration was specified as $100 \mu \mathrm{g}$, where $\mathrm{g}$ is the gravitational acceleration on Earth.

\subsection{Boundary Conditions}

The computational domain extended $15 \mathrm{~d}$ in the axial direction and $6 \mathrm{~d}$ in the radial direction where "d" is the tube inside diameter. These dimensions were established after trials indicating that the near-field flow behavior was independent of the domain size. The upstream boundary was placed at a distance of $1 \mathrm{~d}$ from the jet exit plane to account for the diffusion upstream of the jet exit observed in experimental studies ${ }^{12}$. Symmetric boundary condition was imposed along the jet centerline. At the tube inlet, the velocity profile was fully developed and the helium mole fraction was unity. No slip and zero mass diffusion conditions were imposed at the tube walls. At the exit boundary, pressure outlet condition was implemented where flow properties are 
extrapolated from the interior ${ }^{24}$. Pressure inlet and outlet conditions were specified, respectively, at the upstream and far-field radial boundaries. All flow properties were set to zero at the start of the computations. The operating pressure and temperature were specified, respectively, as $1 \mathrm{~atm}$ and $300 \mathrm{~K}$.

\subsection{Computational Procedure}

An orthogonal non-uniform grid system was used to split the computational domain into five sub regions. The grid elements in the radial direction were concentrated in the flow oscillation region. The grid size was increased gradually in the axial direction. The governing equations were solved sequentially using a segregated approach ${ }^{24}$. The pressure-velocity coupling was achieved using the SIMPLEC algorithm. Grid sensitivity analysis was performed to obtain gridindependent solution. Striking a balance between computational time and accuracy, a grid with 31093 and 80021 nodes was used, respectively, for buoyant and inertial jet analysis. The time step for buoyant and inertial jet computations was $1.6 \mathrm{~ms}$ and $0.3 \mathrm{~ms}$, respectively. The small time step was needed to resolve the high-frequency oscillations in the inertial jet. The numerical code was validated with experimental data ${ }^{12,13}$ and experimental-numerical comparisons are presented for Earth gravity conditions in Ref. 26 and for change from Earth gravity to microgravity in later sections of this paper.

\section{RESULTS AND DISCUSSION}

Time-dependent simulations of the isothermal helium jet injected into the ambient air environment were performed with the objective of quantifying buoyancy effects in the near-field. Although computations were performed for several cases, detailed results are presented for only one buoyant jet and one inertial jet at operating conditions listed in Table 1. First, details 
pertaining to the flow behavior of a buoyant helium jet in Earth gravity and during change from Earth gravity to microgravity are presented. Similar information is provided for the inertial jet. Finally, flow behavior of buoyant and inertial jets is compared in Earth gravity and microgravity.

Table 1. Summary of Test Conditions

\begin{tabular}{|c|c|c|c|c|c|c|}
\hline Case & $R e$ & $R i$ & $d, m m$ & $U_{j}, \mathrm{~m} / \mathrm{s}$ & $\begin{array}{c}f, \mathrm{~Hz} \\
\text { (Computed) }\end{array}$ & $\begin{array}{c}f, \mathrm{~Hz} \\
\text { (Measured) }\end{array}$ \\
\hline Case 1(Buoyant) & 300 & 1.52 & 31.8 & 1.15 & 14.5 & 12.5 \\
\hline Case 2(Inertial) & 800 & 0.018 & 14.05 & 6.75 & 150 & 120 \\
\hline
\end{tabular}

\subsection{Buoyant Jet}

\subsubsection{Flow and Concentration Fields in Earth Gravity}

Figure 1 shows a sequence of helium mole percentage contours superimposed with velocity vectors for the buoyant jet (case 1 ). At $\mathrm{t}=0.0 \mathrm{~ms}$, buoyancy accelerates the jet core, which contracts to conserve the mass. This results in entrainment of ambient fluid producing a toroidal vortex represented by the flow recirculation. Note that the vortex core represented by a black dot is located at $\mathrm{z} / \mathrm{d}=0.12$ in Fig. 1 . In the next plot at $\mathrm{t}=13.6 \mathrm{~ms}$, the vortex is characterized by a larger recirculation region accompanied with greater contraction of the jet core, evident from the inward indentations in the concentration field near $\mathrm{z} / \mathrm{d}=0.2$. Subsequently, between $\mathrm{t}=27.3 \mathrm{~ms}$ and $54.7 \mathrm{~ms}$, the vortex grows in size and convects downstream to contaminate a greater portion of the jet core. The vortex gains its strength by momentum transfer from the jet core. This feature is evident in Fig. 1 wherein the velocity vectors downstream of the vortex core are larger than those upstream before interacting with the jet core. As the vortex propagates downstream, the jet expands near the tube exit inducting buoyant fluid to initiate another vortex at $\mathrm{t}=68.4 \mathrm{~ms}$ to repeat the oscillation cycle. These results illustrate self-excited periodicity in the jet at a frequency of $14.6 \mathrm{~Hz}$, in excellent agreement with the oscillation frequency of $12.5 \mathrm{~Hz}$ measured by Yep et al. ${ }^{13}$ 


\subsubsection{Flow and Concentration Fields during Change from Earth gravity to Microgravity}

Computations were performed to visualize the jet flow during change from Earth gravity to microgravity and to depict how the jet flow adjusts itself in the absence of buoyancy. As a first step, the simulation results were compared with experiments ${ }^{13}$ to validate the computational model. Figure 2 shows a series of computational-experimental helium mole fraction contour plots during change from Earth gravity to microgravity. At the onset of microgravity denoted as $\mathrm{T}=0.0 \mathrm{~s}$, the indentations in the helium concentration level higher than $50 \%$ signify a vortex located at $\mathrm{z} / \mathrm{d}=0.7$. After change to microgravity at $\mathrm{T}=16.66 \mathrm{~ms}$, the vortex convects downstream as the jet expands in the near exit region. At $\mathrm{T}=50.0 \mathrm{~ms}$, the jet has expanded at the exit to $\mathrm{r} / \mathrm{d}=$ 0.7. The indentations in the concentration contours have weakened, signifying the diminishing nature of the vortex. Subsequently, the jet widens gradually throughout the near-field with diminishing oscillations as steady conditions are reached in microgravity. The helium mole fraction contours in microgravity are straight lines reminiscent of a non-buoyant jet. The abovementioned features replicate similar phenomena observed in experiments ${ }^{13}$ showing self-excited oscillations gradually subsiding after microgravity was initiated. Judging from the plots in Figure 2, it is inferred that computations reproduced experimental observations, proving model's capability in predicting the flow structure during change from Earth gravity to microgravity.

Figure 3 shows contours of helium mole percentage overlapped by velocity vectors during change from Earth gravity to microgravity to highlight the interaction of the flow field with the concentration field. At $\mathrm{T}=0.0 \mathrm{~ms}$, a vortex with core located at $\mathrm{z} / \mathrm{d}=0.7$ characterizes the entrainment of ambient fluid into the jet. At this instant the jet flow is similar to that at $\mathrm{T}=27.3 \mathrm{~ms}$ in Fig. 1(c). The jet width characterized by the $10 \%$ helium concentration contour extends to $\mathrm{r} / \mathrm{d}=0.6$ near the exit. In microgravity at $\mathrm{T}=33.33 \mathrm{~ms}$, the jet at the exit has expanded to 
$\mathrm{r} / \mathrm{d}=0.7$. The vortex has convected downstream with its core located at $\mathrm{z} / \mathrm{d}=1.8$. Velocity vectors show a weakening of the vortical structure at this instant. At $\mathrm{T}=66.6 \mathrm{~ms}$, the weakened vortex has moved downstream of the field-of-view while another vortex is formed near the jet exit at $\mathrm{z} / \mathrm{d}=0.3$. At $\mathrm{T}=133.3 \mathrm{~ms}$, flow recirculation has diminished as the jet expands to $\mathrm{r} / \mathrm{d}=0.78$ near the exit. Similar features are exhibited at $\mathrm{t}=166.6 \mathrm{~ms}$ with the flow approaching steady conditions in microgravity. Finally, at $\mathrm{T}=2000 \mathrm{~ms}$ the jet has assumed a steady columnar shape. The flow velocity decreases in the radial direction as the jet fluid mixes with ambient air. Further details about the flow structure are provided by spatio-temporal plots during the change from Earth gravity to microgravity.

\subsubsection{Temporal Evolution of Axial Velocity and Concentration Fields}

Figure 4 depicts temporal evolution of the concentration field during the change from Earth gravity to microgravity. Computed and measured ${ }^{13}$ time traces of helium mole fraction at $\mathrm{z} / \mathrm{d}=1.0$ illustrate that the oscillating jet flow expands radially after the change in gravity. Results show that the oscillations sustain during a brief initial period of microgravity, with steady conditions reached within $\mathrm{T}=0.75 \mathrm{~s}$. Again, the experimental trends are predicted well by the computations. Figure 5 shows time trace plots of helium mole percentage and axial velocity at selected locations during the change from Earth gravity to microgravity. Near the jet exit at $\mathrm{z} / \mathrm{d}=0.15$, the helium concentration at the jet center $(\mathrm{r} / \mathrm{d}=0)$ is constant at $100 \%$ throughout. In contrast, the axial velocity at the jet center fluctuates between $2.3 \mathrm{~m} / \mathrm{s}$ and $2.6 \mathrm{~m} / \mathrm{s}$ in Earth gravity. In microgravity, the axial velocity initially decreases and then reaches a steady value of $2.2 \mathrm{~m} / \mathrm{s}$ at $\mathrm{T}=0.1 \mathrm{~s}$. In the entrainment region at $\mathrm{r} / \mathrm{d}=0.45$, the helium mole percentage in Earth gravity varied between $45 \%$ and $94 \%$. During change to microgravity, the helium concentration peaked to $100 \%$ within 0.08 s. Afterwards, minor oscillations occurred before a steady value of $95 \%$ was 
reached within $\mathrm{T}=0.3 \mathrm{~s}$. Correspondingly, the axial velocity reached a steady value of $0.4 \mathrm{~m} / \mathrm{s}$ in microgravity. Near the jet boundary at $\mathrm{r} / \mathrm{d}=0.6$, low amplitude oscillations in the concentration field are observed in Earth gravity. In microgravity, the helium mole percentage rose to $70 \%$ prior to reaching a steady value of $52 \%$ within $\mathrm{T}=1.0 \mathrm{~s}$. In Earth gravity, the axial velocity profile at this radial location shows troughs with negative velocity indicating flow recirculation.

At a downstream location, $\mathrm{z} / \mathrm{d}=2.0$, pure helium is present throughout at the jet center. The axial velocity and its oscillation amplitude have increased at this location, signifying buoyant acceleration in Earth gravity. The flow oscillations diminish within $0.5 \mathrm{~s}$ of microgravity as steady value of $2.1 \mathrm{~m} / \mathrm{s}$ is reached. At $\mathrm{r} / \mathrm{d}=0.45$, high amplitude concentration oscillations are observed in Earth gravity. At $\mathrm{r} / \mathrm{d}=0.6$, helium reaches peak concentration level of $60 \%$ during change from Earth gravity to microgravity and reaches a steady value of $56 \%$ at $\mathrm{T}=0.6 \mathrm{~s}$. The axial velocity profiles at the above radial locations depict flow behavior similar to that observed upstream. These features agree with time traces of concentration field measured by Yep et al. ${ }^{13}$

\subsection{Inertial Jet}

\subsubsection{Flow and Concentration Fields in Earth Gravity}

Figure 6 shows a sequence of helium mole percentage contours superimposed with velocity vectors to depict an oscillation cycle. In this case, the computed results are presented only between $\mathrm{r} / \mathrm{d}=0.3$ and 0.65 to visualize the flow oscillation region in greater detail. At $\mathrm{t}=0.0 \mathrm{~s}$, buoyant acceleration contracts the jet core leading to entrainment and formation of a toroidal vortex. The flow structure during the oscillation cycle is similar to that of the buoyant jet. The nature and propagation of the toroidal vortex are the same as already explained.

Although self-excited flow oscillations were observed in both buoyant and inertial jets, some aspects of the flow field were found to be different. First, based on the concentration field, the 
inertial jet is wider compared to the buoyant jet. This implies that helium diffuses farther in an inertial jet. This effect is opposite to constant density jets where the jet width decreases with increasing initial jet momentum. Contraction and mixing by entrainment are confined to a narrow region in the inertial case compared to the buoyant case. The oscillation frequency for the inertial jet $(150 \mathrm{~Hz})$ is much higher than that for the buoyant case $(14.5 \mathrm{~Hz})$. The above results illustrate self-excited periodicity in the flow field irrespective of the initial jet momentum, which agrees qualitatively and quantitatively with experiments ${ }^{13,18}$.

\subsubsection{Flow and Concentration Fields during change from Earth gravity to Microgravity}

Figure 7 shows the contours of helium mole percentage overlapped by velocity vectors during change from Earth gravity to microgravity and then to zero gravity to highlight the interaction of flow field with concentration field. At the onset of microgravity $(\mathrm{T}=0.0 \mathrm{~ms})$, a toroidal vortex is located at $\mathrm{z} / \mathrm{d} \cong 0.75$. The vortex convects downstream and a new one is formed near the exit as shown in Fig. 7(b) for $\mathrm{T}=24 \mathrm{~ms}$. Subsequently, the jet flow maintains a periodic structure in microgravity as by Figs. 7(c)-(e) representing an oscillation cycle. The curvature of concentration contours is less severe and the vortex is narrower in microgravity compared to those in Earth gravity. This result is consistent with experiments by Pasumarthi ${ }^{18}$ showing flow oscillations subsiding to smaller amplitudes in microgravity for $\mathrm{Ri}<0.01$. The last plot in Fig 7 pertains to the results obtained in zero gravity, simulated by completely turning off the gravity. In this case, the jet assumes a steady non-oscillatory state to support buoyancy as the cause of the instability. Results show that minor buoyant acceleration destabilizes the inertial jet.

\subsubsection{Temporal Evolution of Axial Velocity and Concentration Fields}

Figure 8 shows time trace plots of helium mole percentage and axial velocity at selected locations during change from Earth gravity to microgravity. At $\mathrm{z} / \mathrm{d}=0.5$, helium concentration at 
the jet centerline remained $100 \%$ throughout. Concentration fluctuations for the inertial case are smaller compared to the buoyant case. Axial velocity at the jet center oscillates between 13 and $14 \mathrm{~m} / \mathrm{s}$ in Earth gravity and between 12.5 and $13.5 \mathrm{~m} / \mathrm{s}$ in microgravity. These oscillations ceased when the gravity was completely turned off in zero gravity. At the downstream location, $\mathrm{z} / \mathrm{d}=2.0$, the axial velocity fluctuates between 12 and $15 \mathrm{~m} / \mathrm{s}$ in Earth gravity. These fluctuations are slightly smaller in microgravity but absent in zero gravity. The concentration and velocity fields in Fig.8 reveal that the flow oscillations subside in microgravity and disappear in zero gravity. These results ascertain that buoyancy plays an important role in both buoyant and inertial lowdensity gas jets.

\subsection{Comparison of Flow Behavior in Buoyant and Inertial Jets}

In this section, buoyant and inertial jet flows are compared to identify similarities and differences in the flow behavior.

\subsubsection{Vortex Convection Velocity}

Figure 9 shows the vortex convection velocity $\left(U_{v}\right)$ normalized by the averaged jet exit velocity in Earth gravity. This plot was generated by tracking the position of the vortex core at different times. Results show a gradual increase of vortex convection velocity in the flow direction. The plots are similar for buoyant and inertial jets although higher values are reached for the buoyant case.

\subsubsection{Axial Velocity Profiles}

As already stated, buoyancy plays an important role in describing the instability phenomena. In case of the buoyant jet, buoyancy effects are prominent in the jet core. However, in the inertial jet, buoyancy affects the low-momentum region of the jet shear layer. This phenomenon is illustrated by the profiles of instantaneous axial velocity at various radial locations as shown in 
Fig.10. The axial velocity was normalized by the jet exit velocity $\left(\mathrm{U}_{\mathrm{e}}\right)$ at that particular radial location. The profiles show the axial velocity varying with axial coordinate and time corresponding to the phases in Figs. 1 and 6.

The normalized axial velocity profiles for the buoyant case (Figs. 10a-c) show periods of acceleration and deceleration at any given phase. The phases reveal the periodic flow behavior of the near-field region. Along the jet centerline, at phase A, slight deceleration occurs up to $\mathrm{z} / \mathrm{d}=0.8$ followed by a rapid acceleration between $\mathrm{z} / \mathrm{d}=1.0$ and 2.0 . Note that the velocity peak at $\mathrm{z} / \mathrm{d}=2.0$ occurs slightly upstream of the vortex core at $\mathrm{z} / \mathrm{d}=2.15$. Near the jet exit, the decelerating flow at phase A accelerates at Phase B as a new vortex is formed. At phase C, rapid acceleration is observed near the exit, with peak normalized axial velocity reaching a value of 1.25 at $\mathrm{z} / \mathrm{d}=0.5$ or slightly upstream of the vortex core at $\mathrm{z} / \mathrm{d}=0.80$. Subsequently, the flow decelerates because of the transfer of the energy from the jet core to the vortex. At later phases (D and E), the flow decelerates near the exit but accelerates at downstream locations. Note that the normalized peak axial velocity has increased to about 1.7 at phase $\mathrm{D}$ and to 2.0 at phase $\mathrm{E}$. A common feature is that the flow accelerates upstream of the vortex core and decelerates downstream of it. The inertial jet shows a similar behavior. However, the normalized axial velocity profiles are elongated implying that the peak occurs farther downstream. The peak normalized axial velocity is within 1.1 for all phases. These results clearly show that the buoyancy induced acceleration at the jet center is more prominent for the buoyant case compared to the inertial case.

At $\mathrm{r} / \mathrm{d}=0.3$, both buoyant and inertial jets exhibit flow acceleration and deceleration because of the interaction with the outer vortex. However, the peak normalized axial velocity has decreased from 2.0 to 1.4 for the buoyant jet and it has increased from 1.1 to 1.2 for the inertial jet. Thus buoyancy is more important at this radial location for the inertial jet. At $r / d=0.45$, both 
buoyant and inertial jets show large variations in the normalized axial velocity because of the small initial momentum in the shear layer region. Note that the flow acceleration is greater for the inertial jet compared to that for the buoyant case.

\subsubsection{Mean and RMS Flow Structure}

The oscillating flow of buoyant and inertial jets is compared in Fig. 11 using the mean and RMS profiles of helium mole percentage and axial velocity at several axial locations. Near the jet exit at $\mathrm{z} / \mathrm{d}=0.05$, the mean concentration shows a top hat profile with finite shear layer thickness for both buoyant and inertial jets. Pure helium is present till $\mathrm{r} / \mathrm{d}=0.38$ for the buoyant case compared to $\mathrm{r} / \mathrm{d}=0.46$ for the inertial case. A steep reduction in helium concentration occurs thereafter. The scalar jet width extends to $\mathrm{r} / \mathrm{d} \cong 0.8$ for the buoyant case and to $\mathrm{r} / \mathrm{d}>0.8$ for the inertial case, indicating greater radial expansion at the higher Reynolds number.

At $\mathrm{z} / \mathrm{d}=0.5$, the mean concentration at the tube inside the boundary plane $(\mathrm{r} / \mathrm{d}=0.5)$ has reduced to $50 \%$ for the buoyant case compared to $75 \%$ for the inertial case, suggesting greater radial mixing for the buoyant case. Further downstream at $\mathrm{z} / \mathrm{d}=1.5$, the concentration profiles show an inflection point located at $\mathrm{r} / \mathrm{d}=0.29$ for the buoyant case and $\mathrm{r} / \mathrm{d}=0.33$ for the inertial case, attributed to the entrainment by the vortex. The concentration gradient in the inflection region is higher for the buoyant case compared to the inertial case. The scalar jet width is nearly independent of the axial location for the buoyant case because the entrainment has restricted radial expansion of the jet. In contrast, for the inertial case, the scalar jet width increases in the flow direction because of the higher jet momentum limiting the entrainment.

The RMS concentration profiles show large fluctuations near the jet exit for the buoyant case. The peak RMS concentration of $14 \%$ at $\mathrm{z} / \mathrm{d}=0.05$ increased to $25 \%$ at $\mathrm{z} / \mathrm{d}=0.5$ and to $29 \%$ at $\mathrm{z} / \mathrm{d}=1.5$. The location of the peak RMS concentration shifts inwards with the axial direction, 
which is consistent with the contraction of the jet as the vortex propagates downstream. The concentration fluctuations confined to a wake region $(0.4<\mathrm{r} / \mathrm{d}<0.6)$ near the jet exit broaden in the flow direction and contaminate a greater portion of the flow at $\mathrm{z} / \mathrm{d}>1.0$. These observations agree with experimental results presented by Pasumarthi. ${ }^{13}$ For the inertial case, fluctuations are negligible at $\mathrm{z} / \mathrm{d}=0.05$. Peak RMS concentration of $10 \%$ at $\mathrm{z} / \mathrm{d}=0.5$ increased to $12 \%$ at $\mathrm{z} / \mathrm{d}=1.5$. Lower fluctuations in the inertial jet compared with buoyant jet signify low level of mixing in the inertial jet.

Mean axial velocity at $\mathrm{z} / \mathrm{d}=0.05$ shows a typical parabolic profile at the tube exit for buoyant and inertial cases. For the buoyant case, the axial velocity at the jet centerline has increased from $2.4 \mathrm{~m} / \mathrm{s}$ at $\mathrm{z} / \mathrm{d}=0.05$ to $2.6 \mathrm{~m} / \mathrm{s}$ at $\mathrm{z} / \mathrm{d}=0.5$ and to $3.1 \mathrm{~m} / \mathrm{s}$ at $\mathrm{z} / \mathrm{d}=1.5$, indicating strong buoyancy induced acceleration. However, the buoyant acceleration is confined mainly to $r / d<0.3$. For the inertial jet case, the mean axial velocity is nearly the same at all axial locations. The RMS axial velocity profiles show negligible fluctuations near the jet exit $(\mathrm{z} / \mathrm{d}=0.05)$ for both cases. The peak fluctuations increase with axial distance. For the buoyant case, peak RMS velocity of $0.3 \mathrm{~m} / \mathrm{s}$ at $\mathrm{z} / \mathrm{d}=0.5$ increased to $0.75 \mathrm{~m} / \mathrm{s}$ at $\mathrm{z} / \mathrm{d}=1.5$. Note that the peak RMS axial velocity shows a decreasing trend from the jet centerline towards the jet boundary plane for the buoyant case. In contrast, the RMS axial velocity peaked at $\mathrm{r} / \mathrm{d} \cong 0.4$ for the inertial case.

\subsubsection{Flow Behavior in Microgravity}

Radial profiles of axial velocity and concentration field in microgravity are presented in Fig. 12 for buoyant and inertial cases. These profiles were obtained after the jet adapted to the change in gravity. In microgravity, the jet assumed steady, non-oscillatory structure for the buoyant case and it maintained flow oscillations for the inertial jet. For both cases, the jet width increased in microgravity. For buoyant jet, the helium concentration at $\mathrm{r} / \mathrm{d}=0.7$ is less than $5 \%$ in Earth 
gravity and approximately $30 \%$ in microgravity. The corresponding values for the inertial jet are between $10-30 \%$ in Earth gravity and between $25-50 \%$ in microgravity. Figure 12 shows that the oscillation amplitude for the inertial jet is significantly smaller in microgravity compared to that in Earth gravity.

\section{CONCLUSIONS}

A laminar, time-dependent, axisymmetric numerical model was developed to simulate helium-air jets previously studied experimentally. The CFD-based model accurately simulated the experiments in Earth gravity and microgravity involving buoyant and inertial jets. The major conclusions of the study are summarized in the following:

- The buoyant jet exhibited self-excited oscillations in Earth gravity at a frequency of 14.5Hz. The inertial jet exhibited similar self-excited oscillations in Earth gravity at a frequency of $150 \mathrm{~Hz}$. However, the oscillation amplitude was smaller and oscillations were confined to a narrow region in the inertial jet compared to the buoyant jet.

- The buoyant jet attained a steady, columnar structure in microgravity. Temporal plots of velocity and concentration fields depict that the flow oscillations persisted for a brief period during the change from Earth gravity to microgravity.

- The inertial jet exhibited self-excited oscillations in microgravity, although the oscillation amplitude was lower. Simulations illustrated that the inertial jet reached a steady, nonoscillatory flow when the gravity was completely turned off (zero gravity). These results show that the inertial jet becomes unstable in the presence of small amount of buoyant acceleration. 
- In Earth gravity, the scalar jet width was found to increase with increasing Reynolds number. This near-field behavior of low-density jets is opposite to that of constant density jets where the jet width decreases at higher Reynolds numbers.

- The scalar jet width increased in microgravity for both buoyant and inertial jets.

\section{ACKNOWLEDGEMENTS}

This work was supported by Physical Sciences Division of NASA's office of Biological and Physical research under grant NAG 3-2388.

\section{REFERENCES}

${ }^{1}$ Fay, J.A., "Buoyant Plumes and Wakes," Annual Review of Fluid Mechanics, Vol. 5, 1973, pp. 151-160.

${ }^{2}$ Morton, B.A., "Forced Plumes,” Journal of Fluid Mechanics, Vol. 5, 1959, pp. 151-159.

${ }^{3}$ List, E.L., "Turbulent Jets and Plumes," Annual Review of Fluid Mechanics, Vol. 14, 1982, pp. 189-212.

${ }^{4}$ Papanicolaou, P.A., and List, E.L., "Investigations of Round Vertical Turbulent Buoyant Jets," Journal of Fluid Mechanics, Vol. 195, 1988, pp. 342-391.

${ }^{5}$ Dai, Z., Tseng, L.K., and Faeth G.M., "Structure of Round, Fully Developed, Buoyant Turbulent Plumes," Journal of Heat Transfer, Vol. 116, 1994, pp. 409-417.

${ }^{6}$ So, R.M.C., Zhu, J.Y., Otugen, M.V., and Hwang, B.C., "Some Measurements in a Binary Gas Jet," Experiments in Fluids, Vol. 9, 1990, pp. 273-284.

${ }^{7}$ Subbarao, E.R., and Cantwell B.J., "Investigation of a Co-Flowing Buoyant Jet: Experiments on the Effects of Reynolds number and Richardson number," Journal of Fluid Mechanics, Vol.245, 1992, pp. 69-90.

${ }^{8}$ Hamins, A., Yang, J.C., and Kashiwagi, T., "An Experimental Investigation of the Pulsating Frequency of Flames," Proceedings of the Combustion Institute Vol. 24, 1992, pp. 1695-1705.

${ }^{9}$ Cetegen, B.M., and Ahmed, T.A., "Experiments on the Periodic Instability of Buoyant Plumes and Pool Fires," Combustion and Flame, Vol. 93, 1993, pp. 157-184. 
${ }^{10}$ Cetegen, B.M., and Kasper, K.D., "Experiments on the Oscillatory Behavior of Buoyant Plumes of Helium and Helium-Air Mixtures," Physics of Fluids, Vol. 8, 1996, pp. 2974-2984.

${ }^{11}$ Cetegen, B.M., "Measurements of Instantaneous Velocity Field of a Non-Reacting Pulsating Buoyant Plume by Particle Image Velocimetry," Combustion Science and Technology, Vol. 123, 1997, pp. 377-387.

${ }^{12}$ Pasumarthi, K.S., and Agrawal, A.K., "Schlieren Measurements and Analysis of Concentration Field in Self-Excited Helium Jets," Physics of Fluids, Vol. 15, 2003, pp. 3683-3692.

${ }^{13}$ Yep, T.W., and Agrawal, A.K., "Gravitational Effects on Near-Field Flow Structure of Low Density Gas Jets,” AIAA Journal, Vol. 41, 2003, pp. 1973-1979.

${ }^{14}$ Sreenivasan, K.R., Raghu, S., and Kyle, D.M., “Absolute Instability in Variable Density Round Jets,” Experiments in Fluids, Vol. 7, 1989, pp. 309-317.

${ }^{15}$ Monkewitz, P.A., Bechert, D.W., Bariskov, B., and Lehmann, B., "Self Excited Oscillations and Mixing in a heated Round Jet," Journal of Fluid Mechanics, Vol. 213, 1990, pp. 611-639.

${ }^{16}$ Kyle, D.M., and Sreenivasan, K.R., "The Stability and Breakdown of a Round Variable Density Jet," Journal of Fluid Mechanics, Vol. 249, 1993, pp. 619-664.

${ }^{17}$ Richards,C.D.,Breuel,B.D.,Clark, R.P., and Troutt, T.R., "Concentration Measurements in a Self-Excited Jet," Experiments in Fluids, Vol. 21,1996, pp. 103-109.

${ }^{18}$ Pasumarthi, K.S and Agrawal, A.K., "Buoyancy Effects on Flow Structure and Instability of Low-Density Gas Jets,” Doctoral Dissertation, University of Oklahoma, 2004.

${ }^{19}$ Mell, W.E., McGrattan, W.B., and Baum, H.R., "Numerical Simulation of Combustion in Fire Plumes," Proceedings of the Combustion Institute, Vol. 26, 1996, pp. 1523-1530.

${ }^{20}$ Soteriou, M.C., Dong, Y., and Cetegen, B.M., "Lagrangian Simulation of the Unsteady Nearfield Dynamics of Planar Buoyant Plumes," Physics of Fluids, Vol. 14, 2002, pp. 3118-3140.

${ }^{21}$ Katta, V.R., and Goss, L.P., "Numerical Investigations of Transitional $\mathrm{H}_{2} / \mathrm{N}_{2}$ Jet Diffusion Flames," AIAA Journal, Vol. 32, 1994, pp. 84-94.

${ }^{22}$ Sato, H., Kushida, G., Amagai, K., and Arai, M., "Numerical Analysis of the Gravitational Effect on the Buoyancy-Driven Fluctuations in Diffusion Flames," Proceedings of the Combustion Institute, Vol. 29, 2002, pp. 1671-1678.

${ }^{23}$ Bird, R.B., Stewart, W.E., Lightfoot, E.N., “Transport Phenomena, 1 st edition, 1960, Wiley, New York.

${ }^{24}$ Fluent 6.0, User's Guide, Fluent Inc. Lebanon, NH 1-4 (2003). 
${ }^{25}$ Gambit 2.1, User's Guide, Fluent Inc. Lebanon, NH 1-4 (2003).

${ }^{26}$ Satti, R.P., and Agrawal, A.K., "Numerical Analysis of Flow Evolution in a Helium Jet injected into Ambient Air," ASME Paper No. HTFED-2004-56811.

${ }^{27}$ Satti, R.P., Pasumarthi, K.S., and Agrawal, A.K., "Numerical Simulations of Buoyancy Effects in Low Density Gas Jets," AIAA Paper-2004-1317 


\section{LIST OF FIGURES}

Figure 1. Contours of helium mole percentage superimposed by velocity vectors during an oscillation cycle in Earth gravity for $\mathrm{Re}=300, \mathrm{Ri}=1.52$, and $\mathrm{d}=31.8 \mathrm{~mm}$. (a) $\mathrm{t}=0.0 \mathrm{~ms}$; (b) $\mathrm{t}=13.6 \mathrm{~ms}$; (c) $\mathrm{t}=27.3 \mathrm{~ms}$; (d) $\mathrm{t}=41.0 \mathrm{~ms}$; (e) $\mathrm{t}=54.7 \mathrm{~ms}$; (f) $\mathrm{t}=68.4 \mathrm{~ms}$.

Figure 2. Measured (1) and Computed (2) helium mole fraction contours during change from Earth gravity to microgravity. $\mathrm{Re}=300, \mathrm{Ri}=1.52$, and $\mathrm{d}=31.8 \mathrm{~mm}$. (a) $\mathrm{T}=0.0 \mathrm{~ms}$; (b) $\mathrm{T}=16.66 \mathrm{~ms}$; (c) $\mathrm{T}=50.00 \mathrm{~ms}$; (d) $\mathrm{T}=216.66 \mathrm{~ms}$; (e) $\mathrm{T}=2000 \mathrm{~ms}$.

Figure 3. Contours of helium mole percentage superimposed by velocity vectors during change from Earth gravity to microgravity for $\mathrm{Re}=300, \mathrm{Ri}=1.52$, and $\mathrm{d}=31.8 \mathrm{~mm}$ (a) $\mathrm{T}=0.0 \mathrm{~ms}$; (b) $\mathrm{T}=33.33 \mathrm{~ms}$; (c) $\mathrm{T}=66.66 \mathrm{~ms}$; (d) $\mathrm{T}=133.33 \mathrm{~ms}$; (e) $\mathrm{T}=166.66 \mathrm{~ms}$; (f) $\mathrm{T}=2000 \mathrm{~ms}$.

Figure 4. Measured (a) and Computed (b) time traces of helium mole fraction during change from Earth gravity to microgravity. $\mathrm{Re}=300, \mathrm{Ri}=1.52$ and $\mathrm{d}=31.8 \mathrm{~mm}$ at $\mathrm{z} / \mathrm{d}=1.0$.

Figure 5. Time traces of 1) helium mole percentage and 2) axial velocity during change from Earth gravity to microgravity for $\mathrm{Re}=300, \mathrm{Ri}=1.52$ and $\mathrm{d}=31.8 \mathrm{~mm}$. a) $\mathrm{z} / \mathrm{d}=0.15 \mathrm{~b}$ ) $\mathrm{z} / \mathrm{d}=2.0$.

Figure 6. Contours of helium mole percentage superimposed by velocity vectors during an oscillation cycle in Earth gravity for $\mathrm{Re}=800, \mathrm{Ri}=0.018$ and $\mathrm{d}=14.05 \mathrm{~mm}$. (a) $\mathrm{t}=0.0 \mathrm{~ms}$; (b) $\mathrm{t}=1.2 \mathrm{~ms}$; (c) $\mathrm{t}=2.4 \mathrm{~ms}$; (d) $\mathrm{t}=3.6 \mathrm{~ms}$; (e) $\mathrm{t}=4.8 \mathrm{~ms}$; (f) $\mathrm{t}=6.6 \mathrm{~ms}$.

Figure 7. Contours of helium mole percentage superimposed by velocity vectors during change from Earth gravity to microgravity for $\mathrm{Re}=800, \mathrm{Ri}=0.018$ and $\mathrm{d}=14.05 \mathrm{~mm}$. (a) $\mathrm{T}=0.0 \mathrm{~ms}$; (b) $\mathrm{T}=24 \mathrm{~ms}$; (c) $\mathrm{T}=400 \mathrm{~ms}$; (d) $\mathrm{T}=402.5 \mathrm{~ms}$; (e) $\mathrm{T}=405.8 \mathrm{~ms}$; (f) zero gravity.

Figure 8. Time traces of 1) helium mole percentage and 2) axial velocity during change from Earth gravity to micro gravity for $\mathrm{Re}=800, \mathrm{Ri}=0.018$ and $\mathrm{d}=14.05 \mathrm{~mm}$ at a) $\mathrm{z} / \mathrm{d}=0.5 \mathrm{~b}$ ) $\mathrm{z} / \mathrm{d}=2.0$.

Figure 9. Normalized Vortex Convection velocities for $\mathrm{Re}=300, \mathrm{Ri}=1.52$ and $\mathrm{d}=31.8 \mathrm{~mm}$ and $\mathrm{Re}=800, \mathrm{Ri}=0.018$ and $\mathrm{d}=14.05 \mathrm{~mm}$ cases.

Figure 10. Normalized axial velocity for 1) $\mathrm{Re}=300$ and 2) $\mathrm{Re}=800$ at different radial locations of a) $r / d=0.0 \mathrm{~b}$ ) $\mathrm{r} / \mathrm{d}=0.3 \mathrm{c}) \mathrm{r} / \mathrm{d}=0.45$.

Figure 11. Mean and RMS helium mole percentage and axial velocity profiles at different axial stations for a) $\mathrm{Re}=300, \mathrm{Ri}=1.52$, and $\mathrm{d}=31.8 \mathrm{~mm}$ and $\mathrm{b}$ ) $\mathrm{Re}=800, \mathrm{Ri}=0.018, \mathrm{~d}=14.05 \mathrm{~mm}$ in Earth gravity.

Figure 12. Helium mole percentage and axial velocity profiles at different axial stations for a) $\mathrm{Re}=300, \mathrm{Ri}=1.52$, $\mathrm{d}=31.8 \mathrm{~mm}$ and $\mathrm{b}$ ) $\mathrm{Re}=800, \mathrm{Ri}=0.018, \mathrm{~d}=14.05 \mathrm{~mm}$ cases in microgravity. 


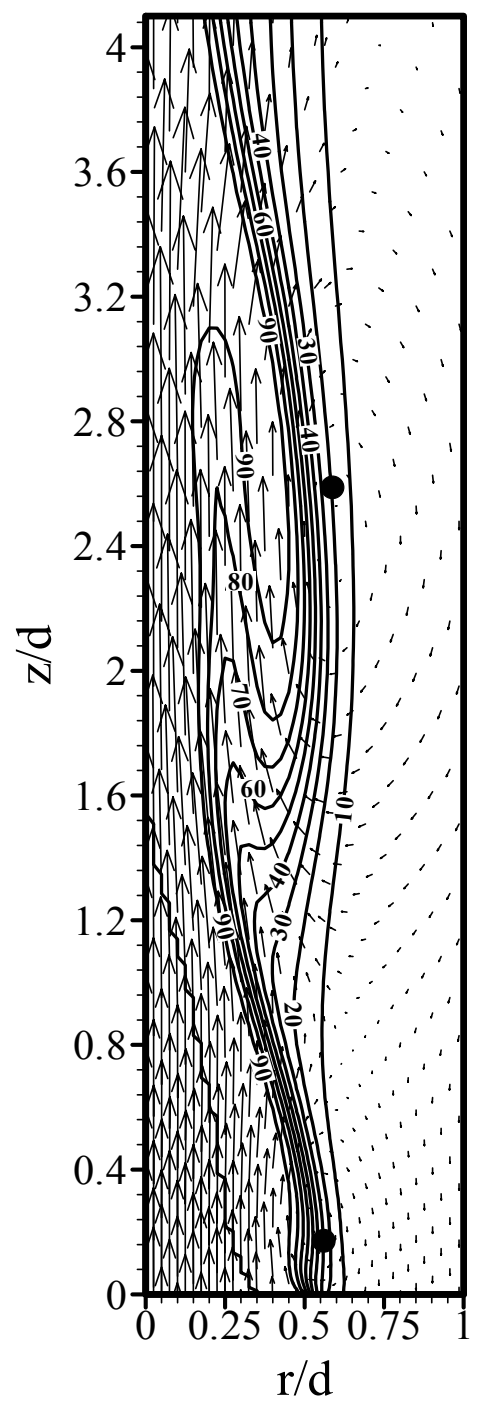

(a)

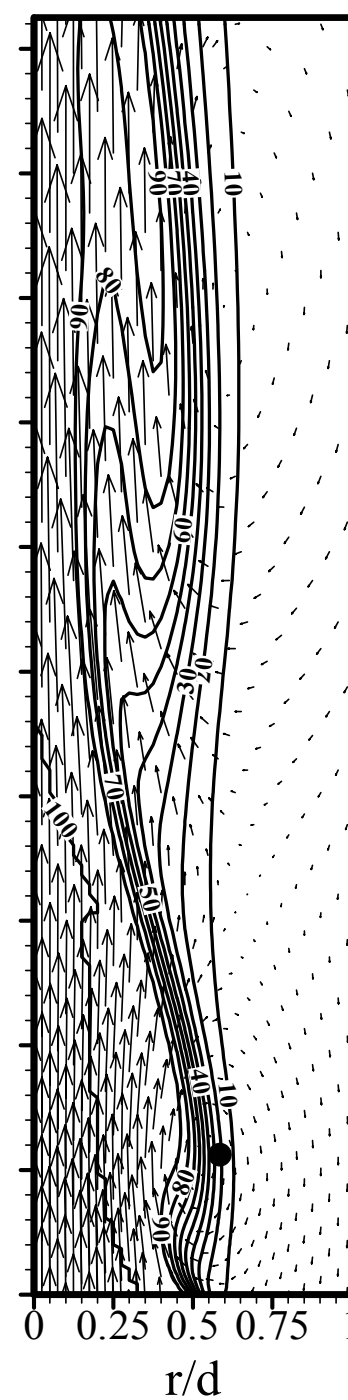

(b)

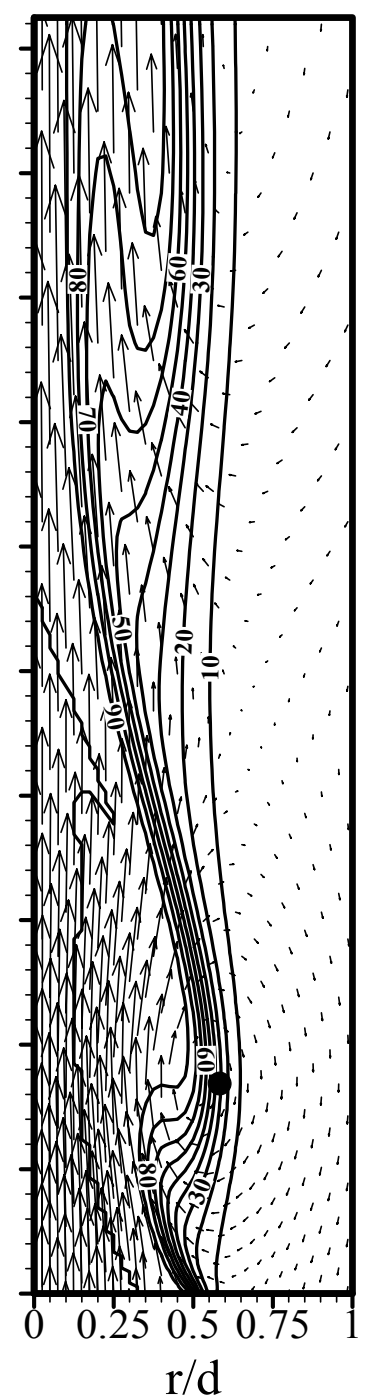

(c)

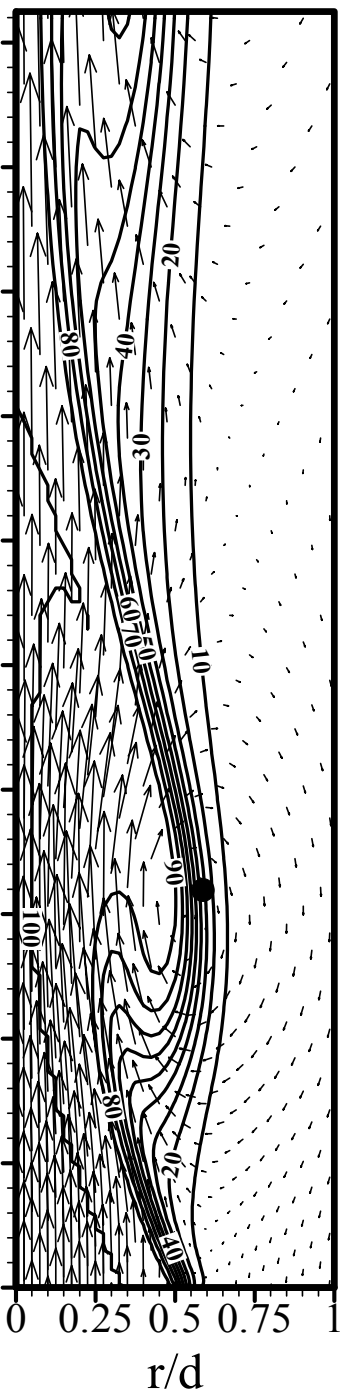

(d)

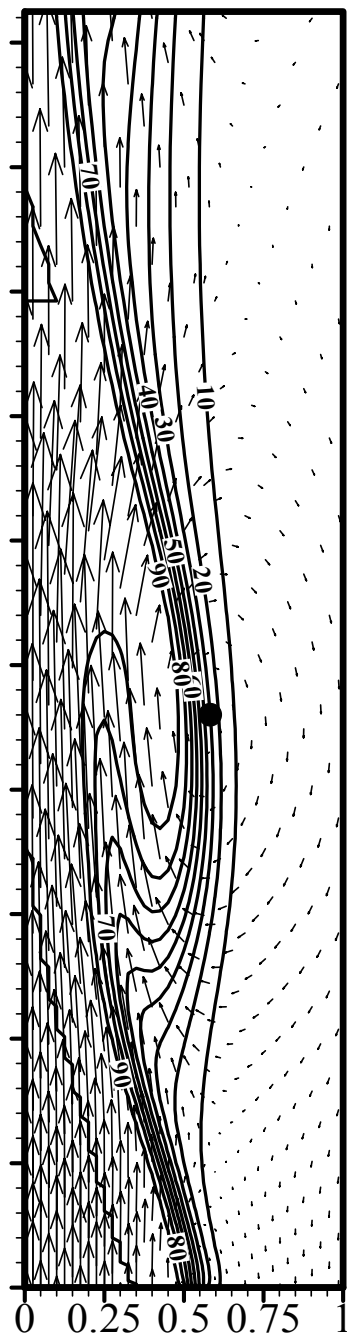

$\mathrm{r} / \mathrm{d}$

(e)

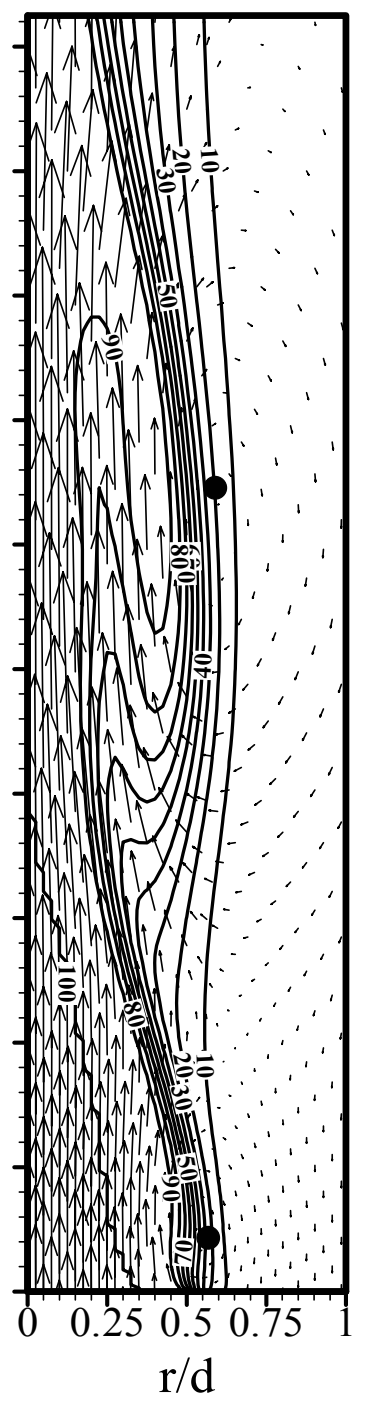

(f)

Fig.1, $A I A A$ 

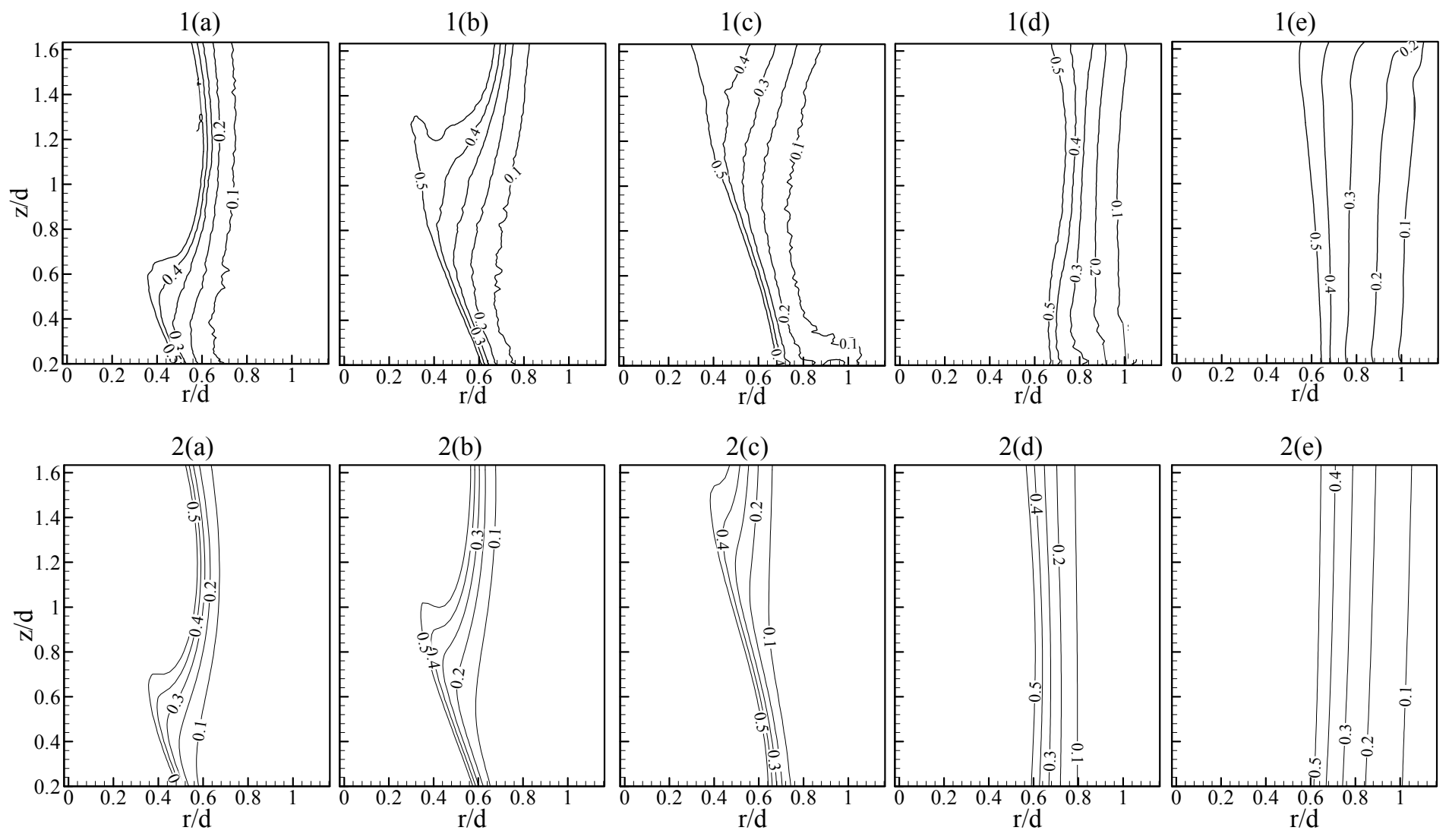

Fig. 2, AIAA 


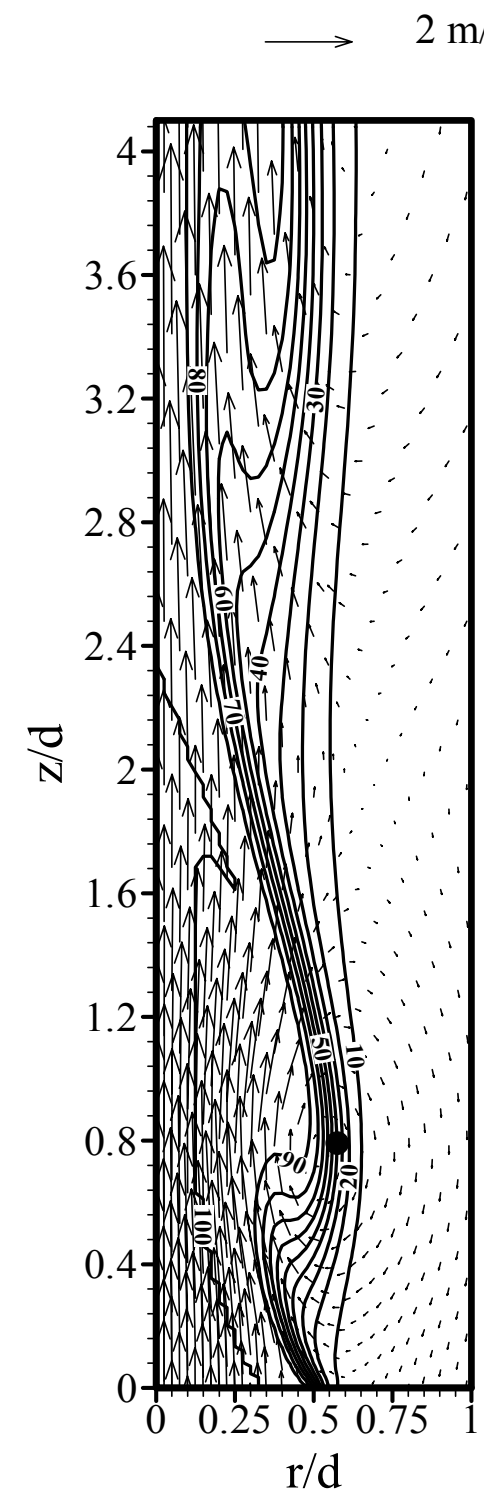

(a)

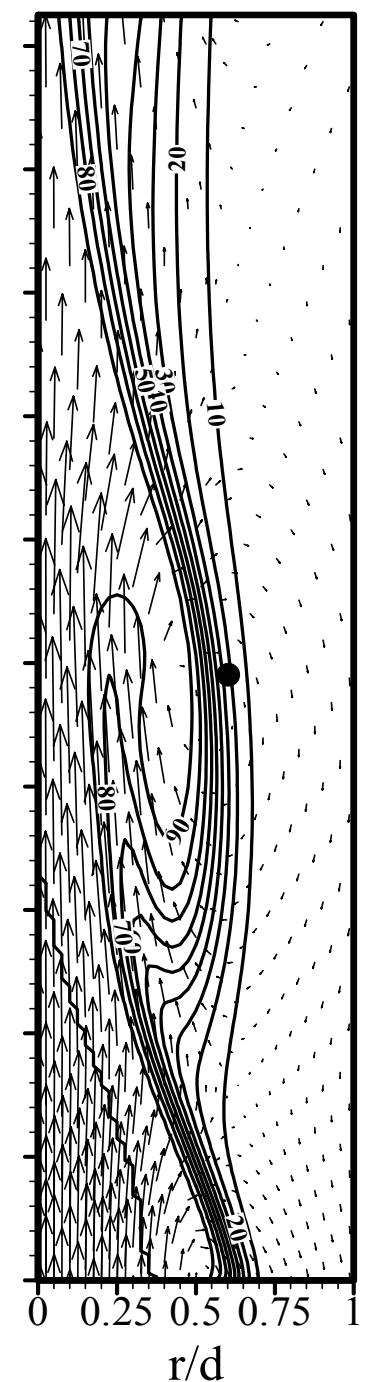

(b)

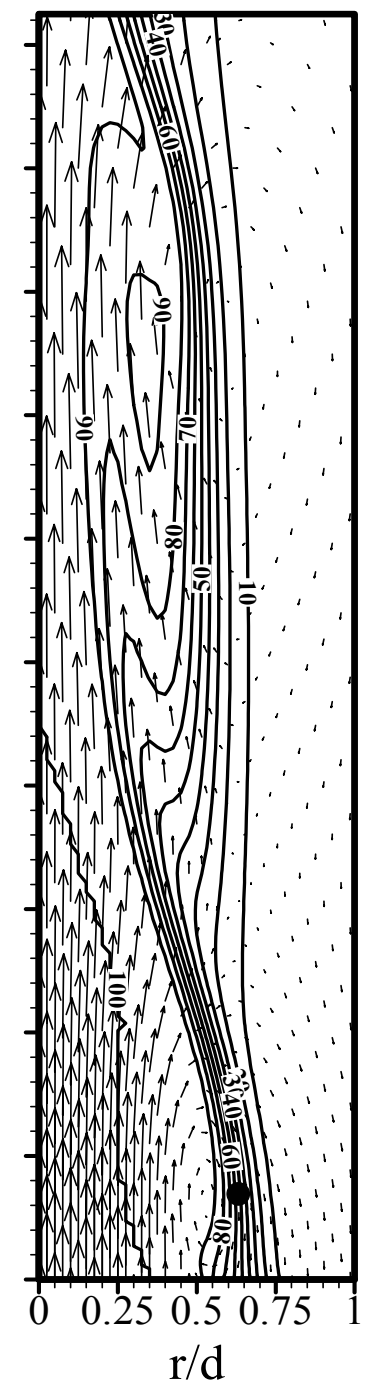

(c)

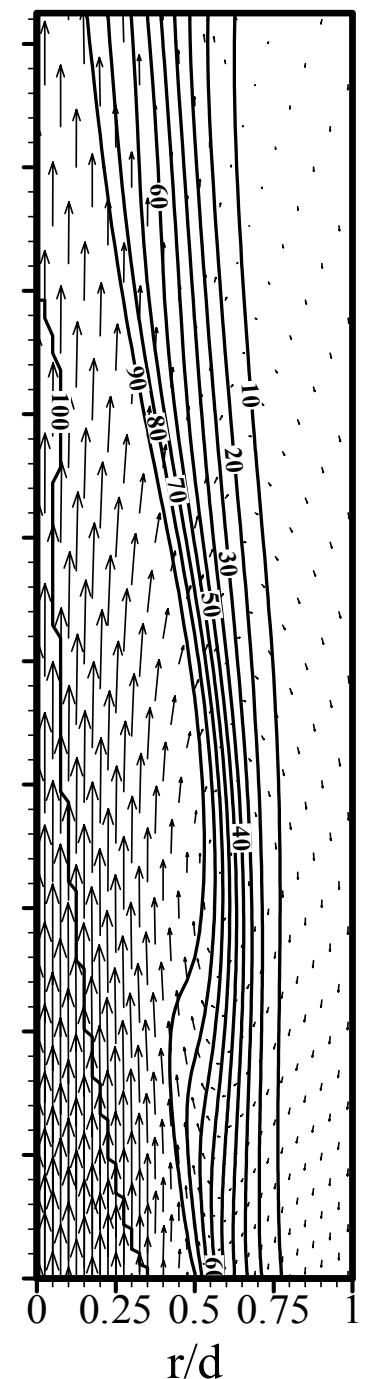

(d)

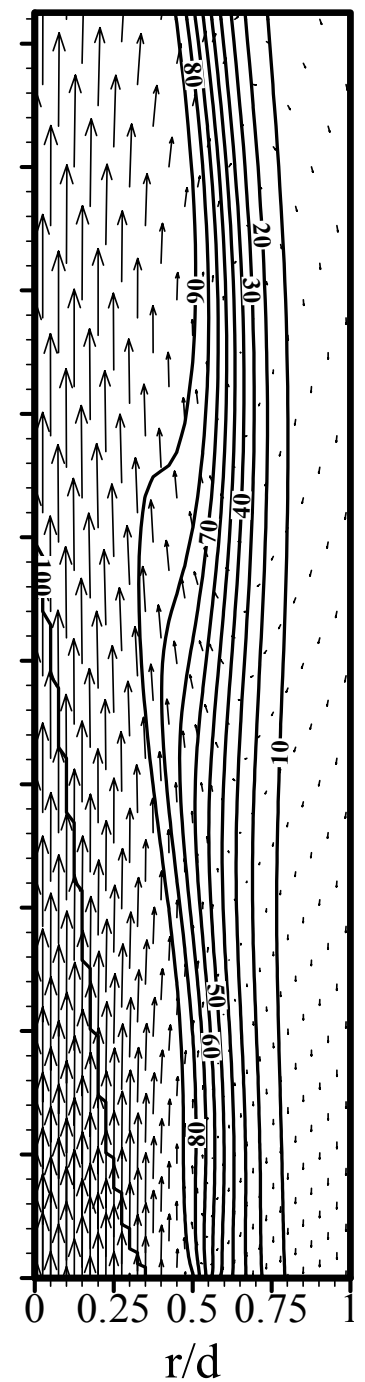

(e)

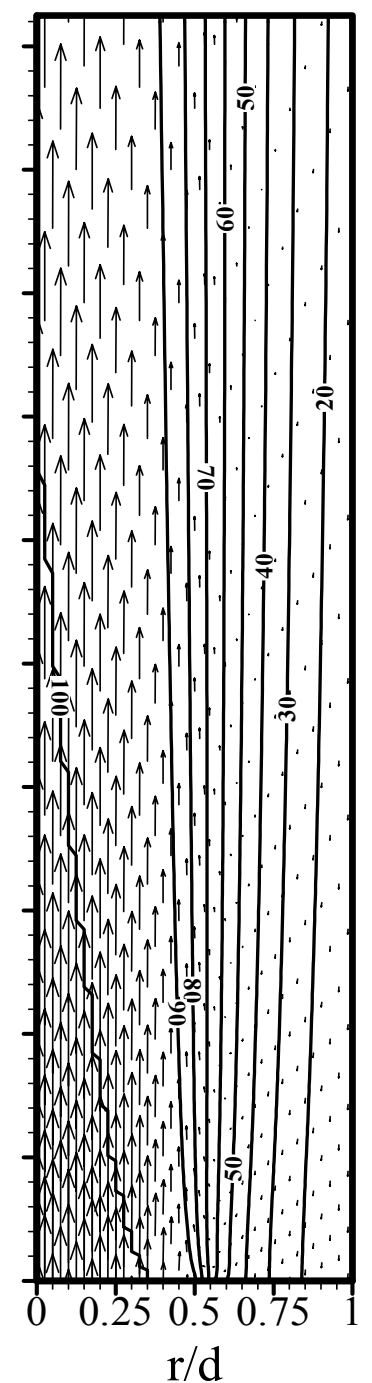

(f)

Fig . 3, AIAA 


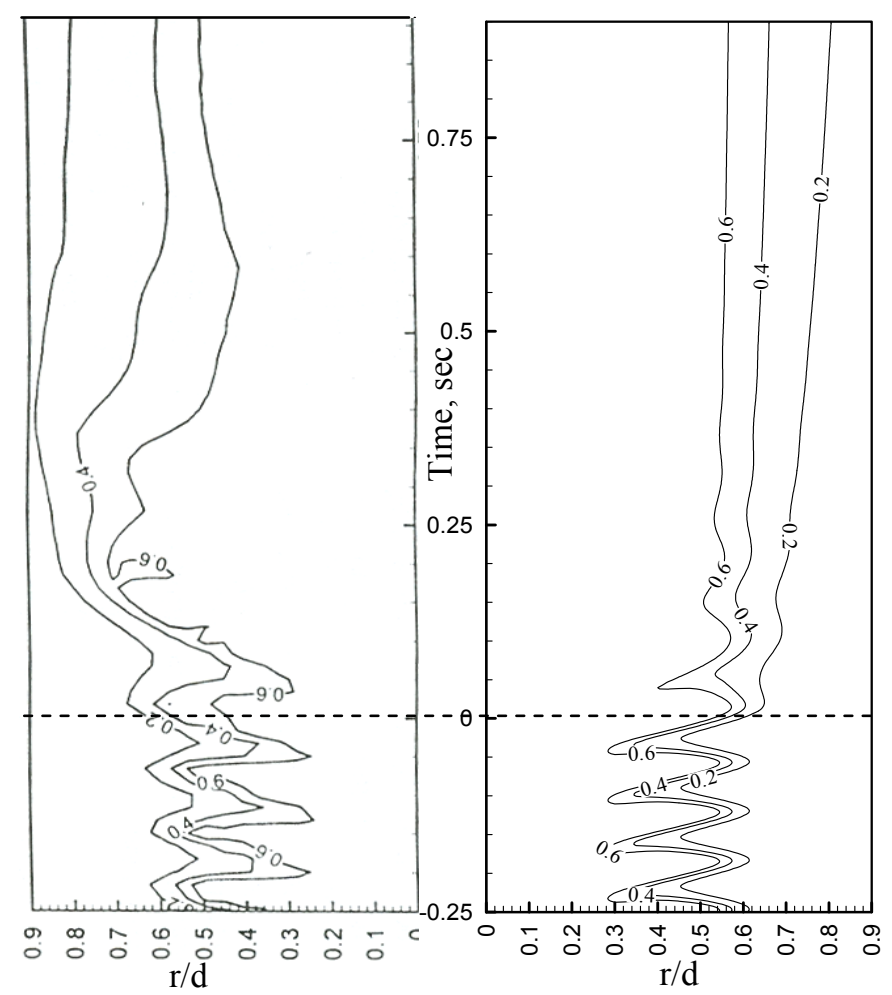

(a)

(b)

Fig. 4, AIAA 


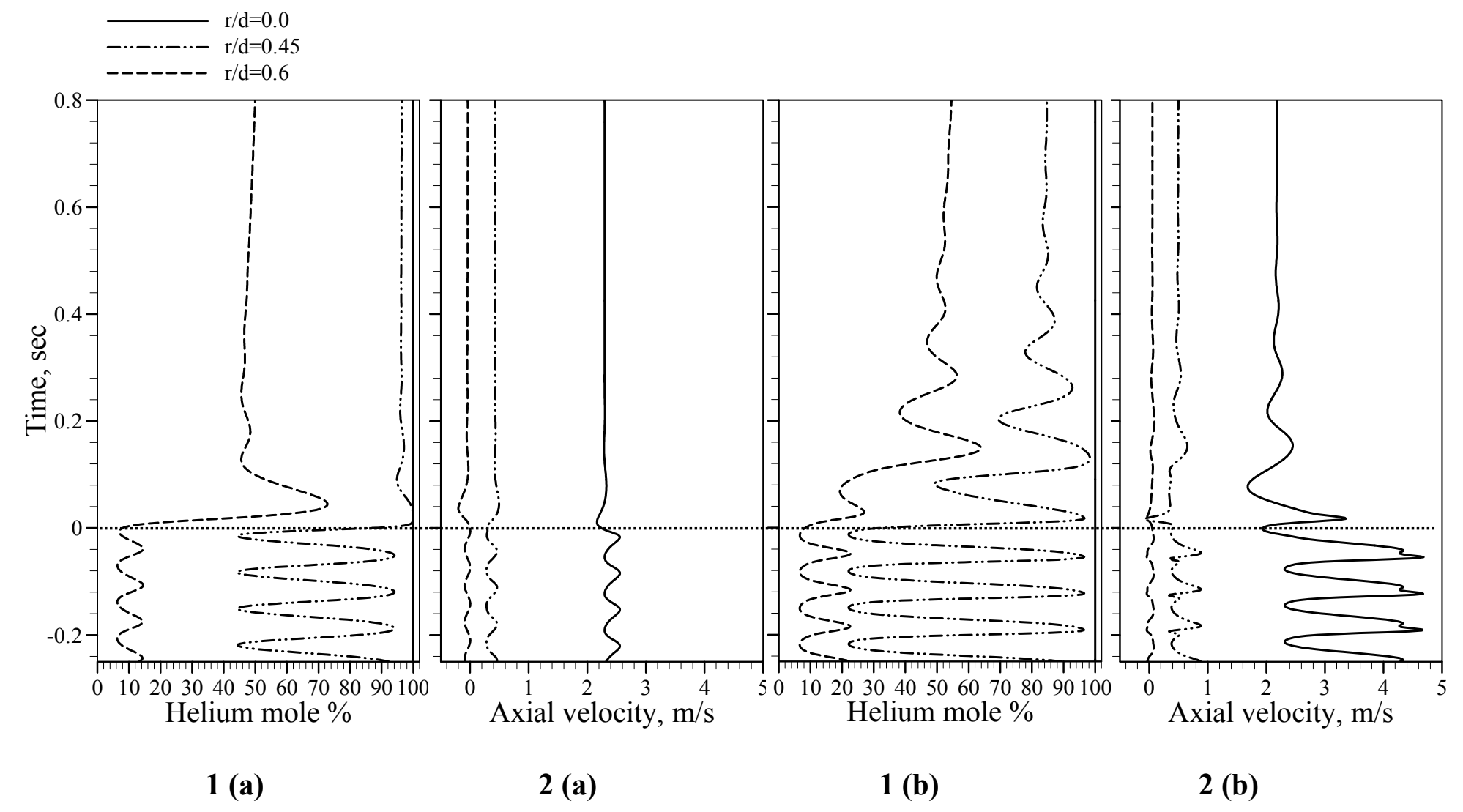

Fig. 5, AIAA 

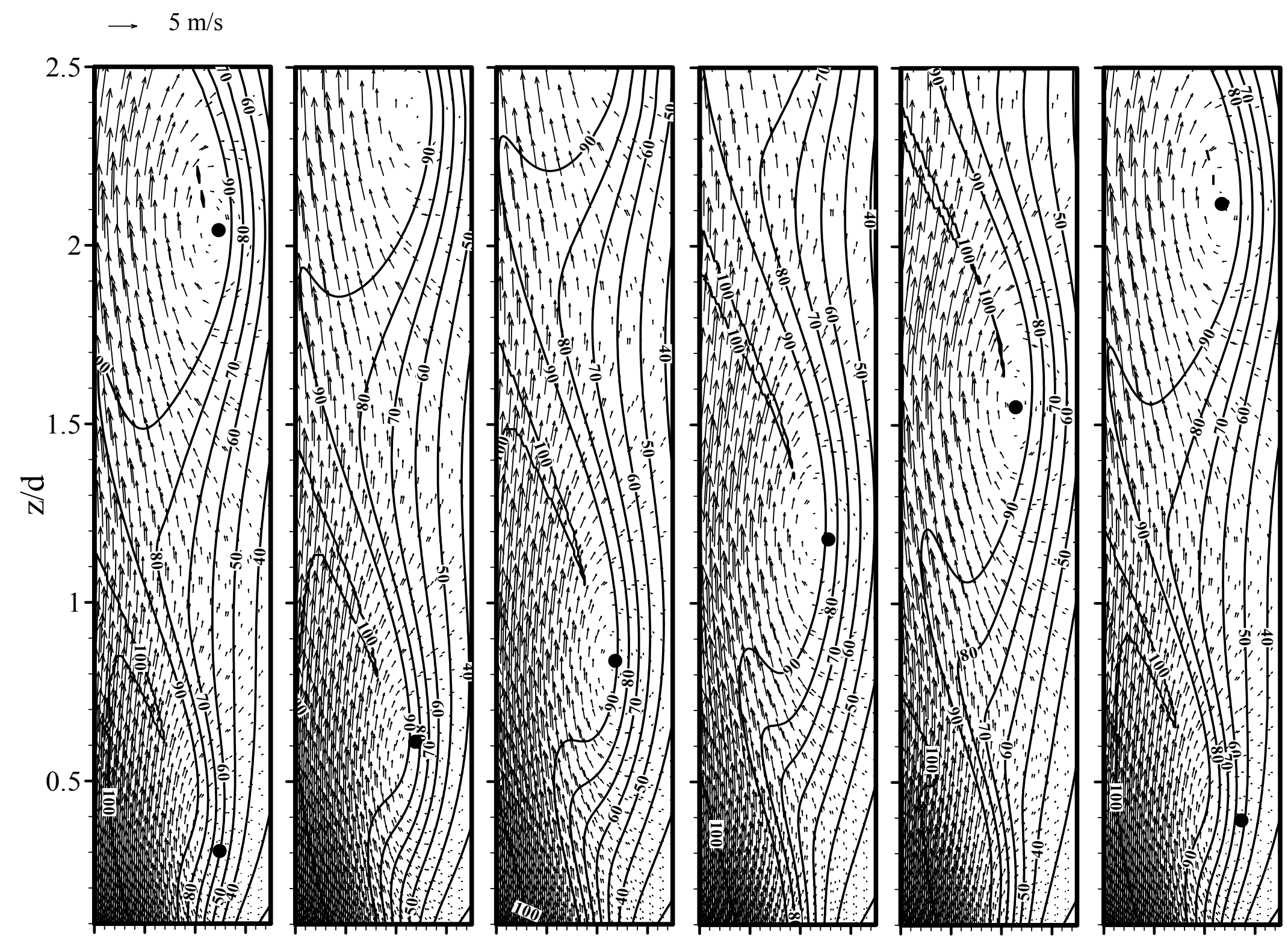

$\begin{array}{llllllllllllllllllllllll}0.3 & 0.4 & 0.5 & 0.6 & 0.3 & 0.4 & 0.5 & 0.6 & 0.3 & 0.4 & 0.5 & 0.6 & 0.3 & 0.4 & 0.5 & 0.6 & 0.3 & 0.4 & 0.5 & 0.6 & 0.3 & 0.4 & 0.5 & 0.6\end{array}$ $\mathrm{r} / \mathrm{d}$

(a)

(b)

(c)

$\mathrm{r} / \mathrm{d}$

(d) $\mathrm{r} / \mathrm{d}$

(e) $\mathrm{r} / \mathrm{d}$

(f)

Fig. 6, AIAA 

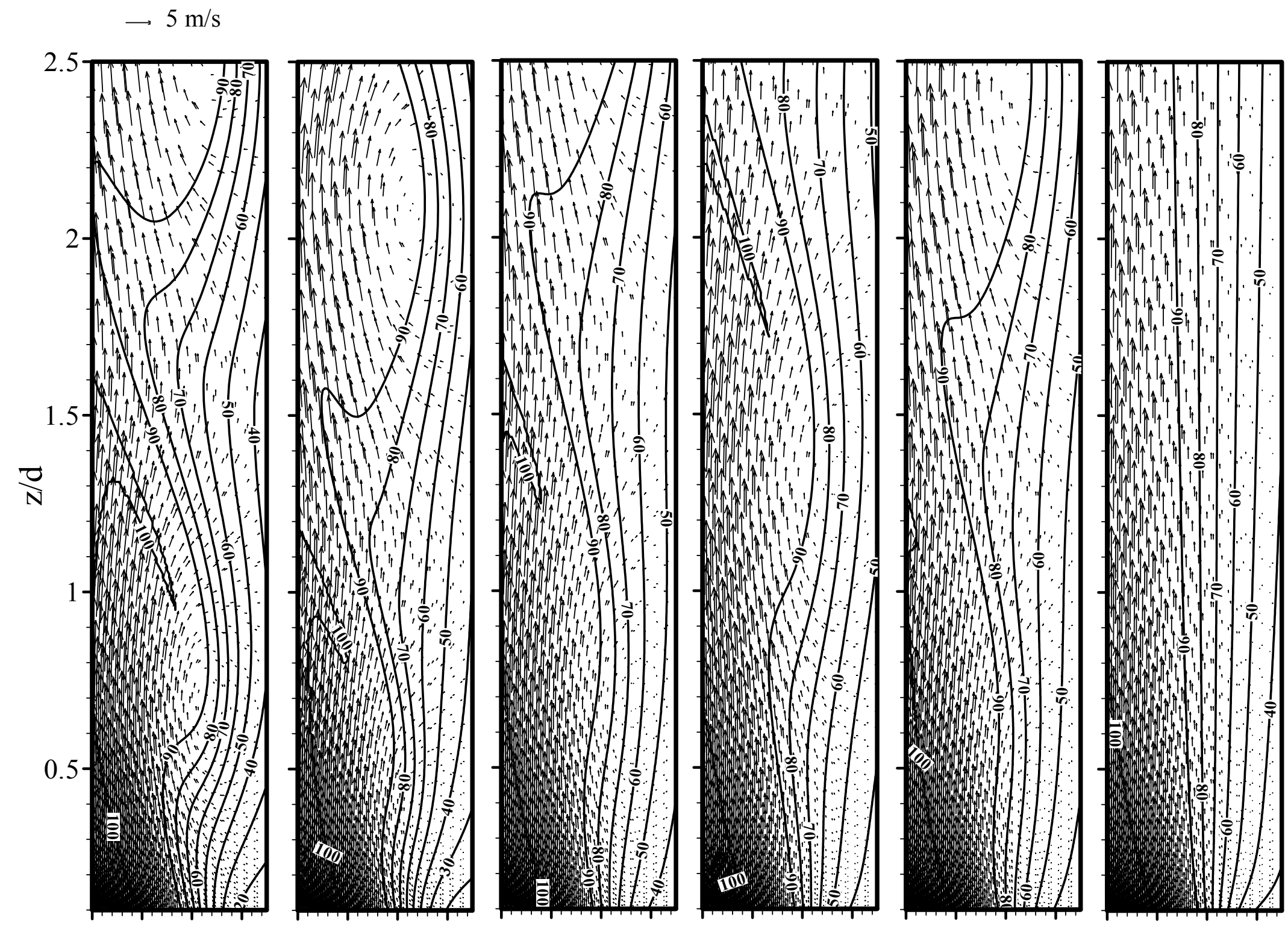

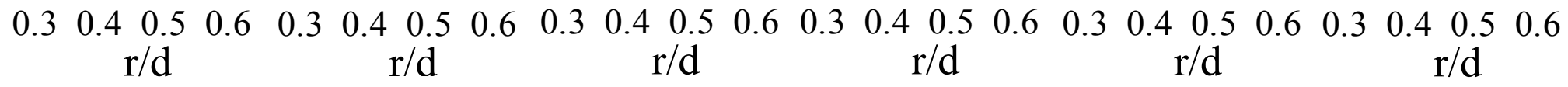

(a)

(b)

(c)

(d)

(e)

(f)

Fig. 7, AIAA 


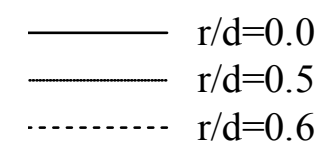

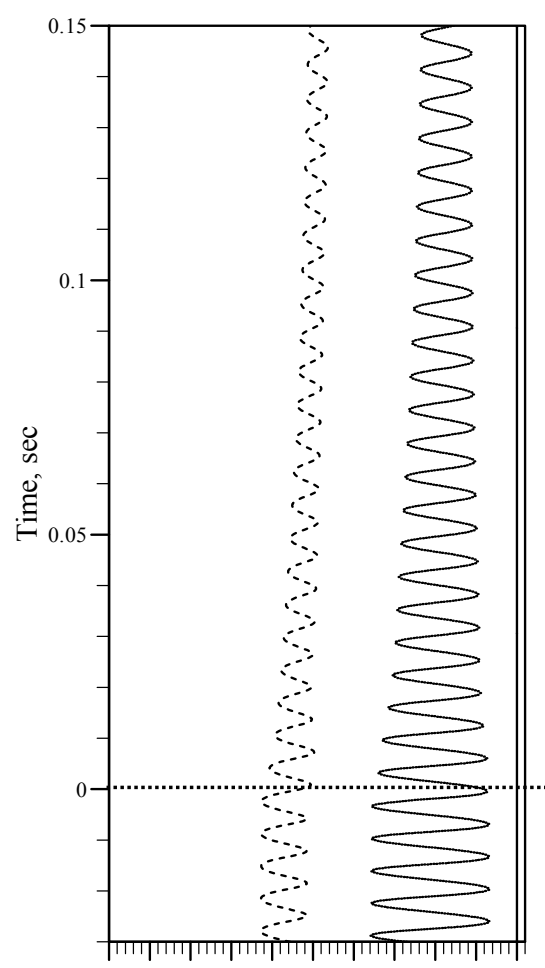

$\begin{array}{lllllllllll}0 & 10 & 20 & 30 & 40 & 50 & 60 & 70 & 80 & 90 & 100\end{array}$

Helium mole \%

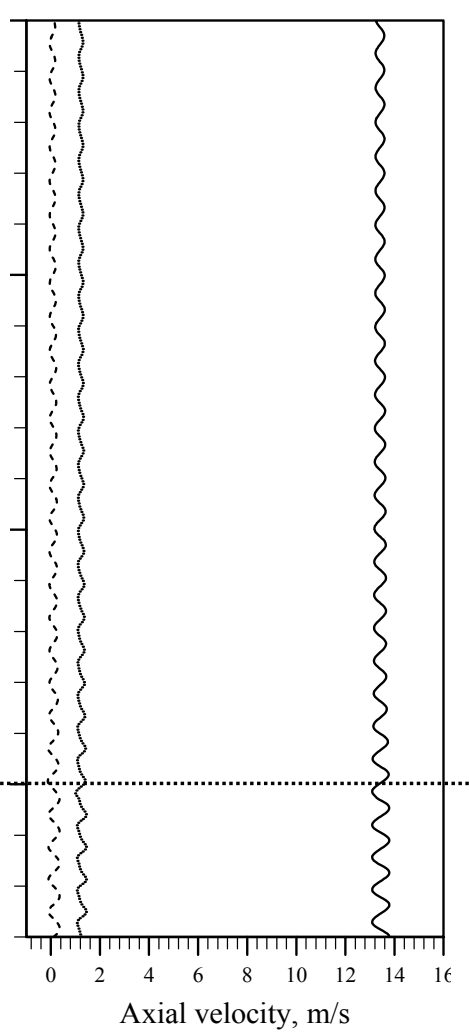

Axial velocity, $\mathrm{m} / \mathrm{s}$

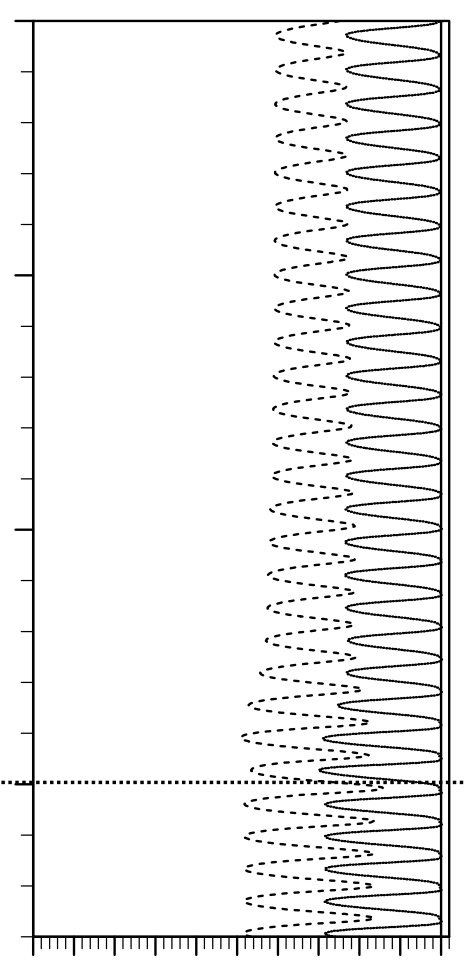

$\begin{array}{lllllllllll}0 & 10 & 20 & 30 & 40 & 50 & 60 & 70 & 80 & 90 & 100\end{array}$ Helium mole \%

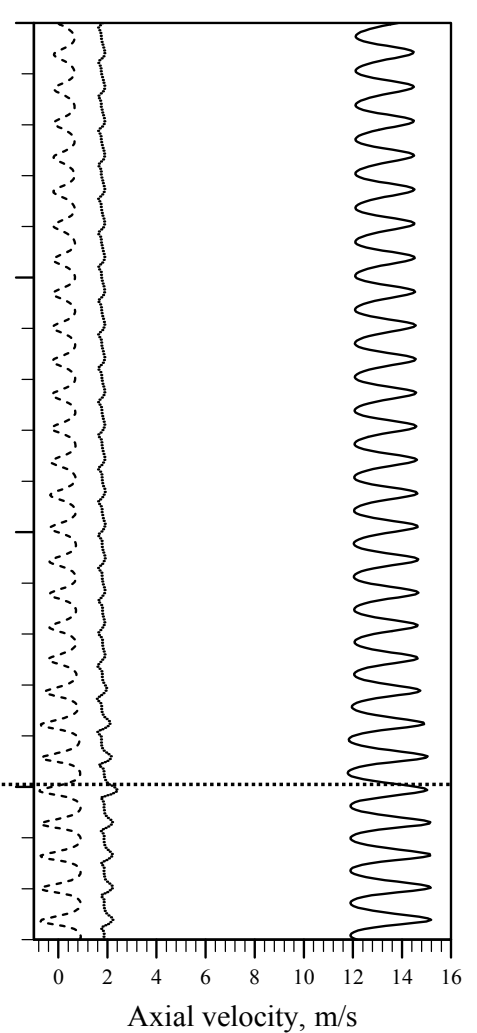

2 (b)

Fig. 8, AIAA 


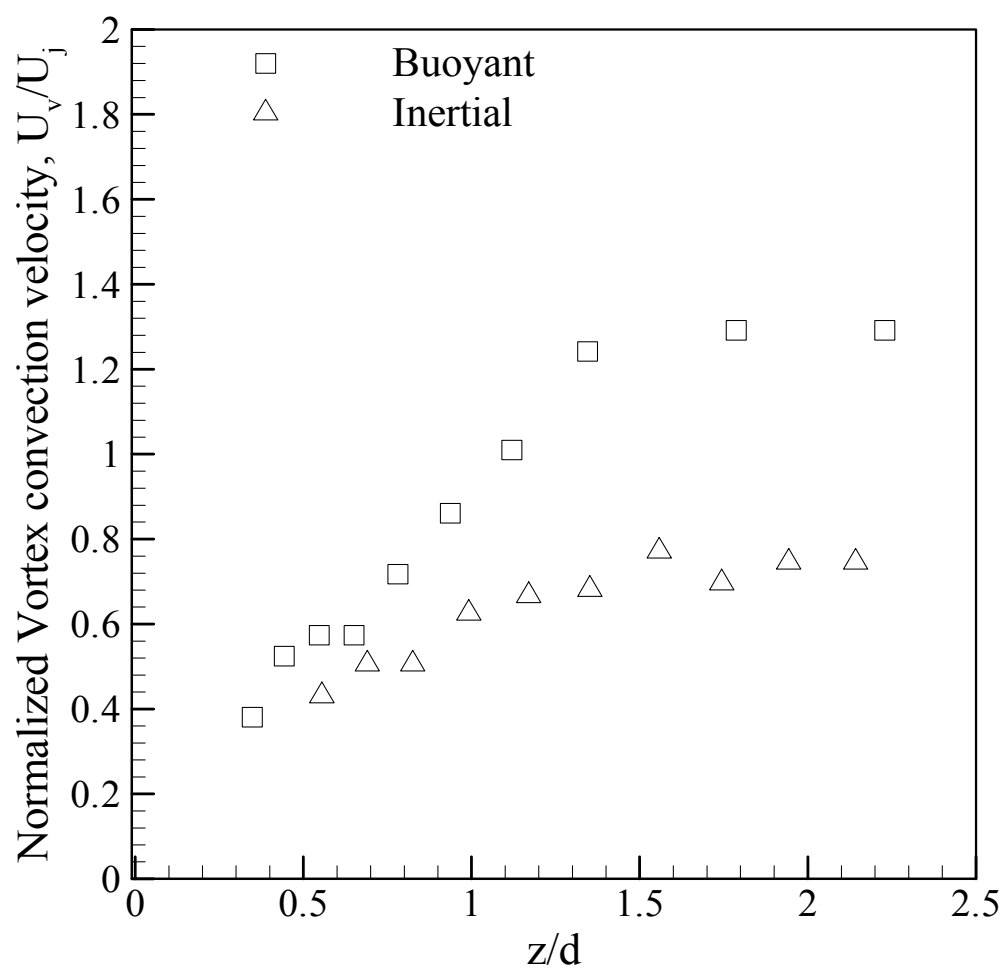

Fig. 9, $A I A A$ 

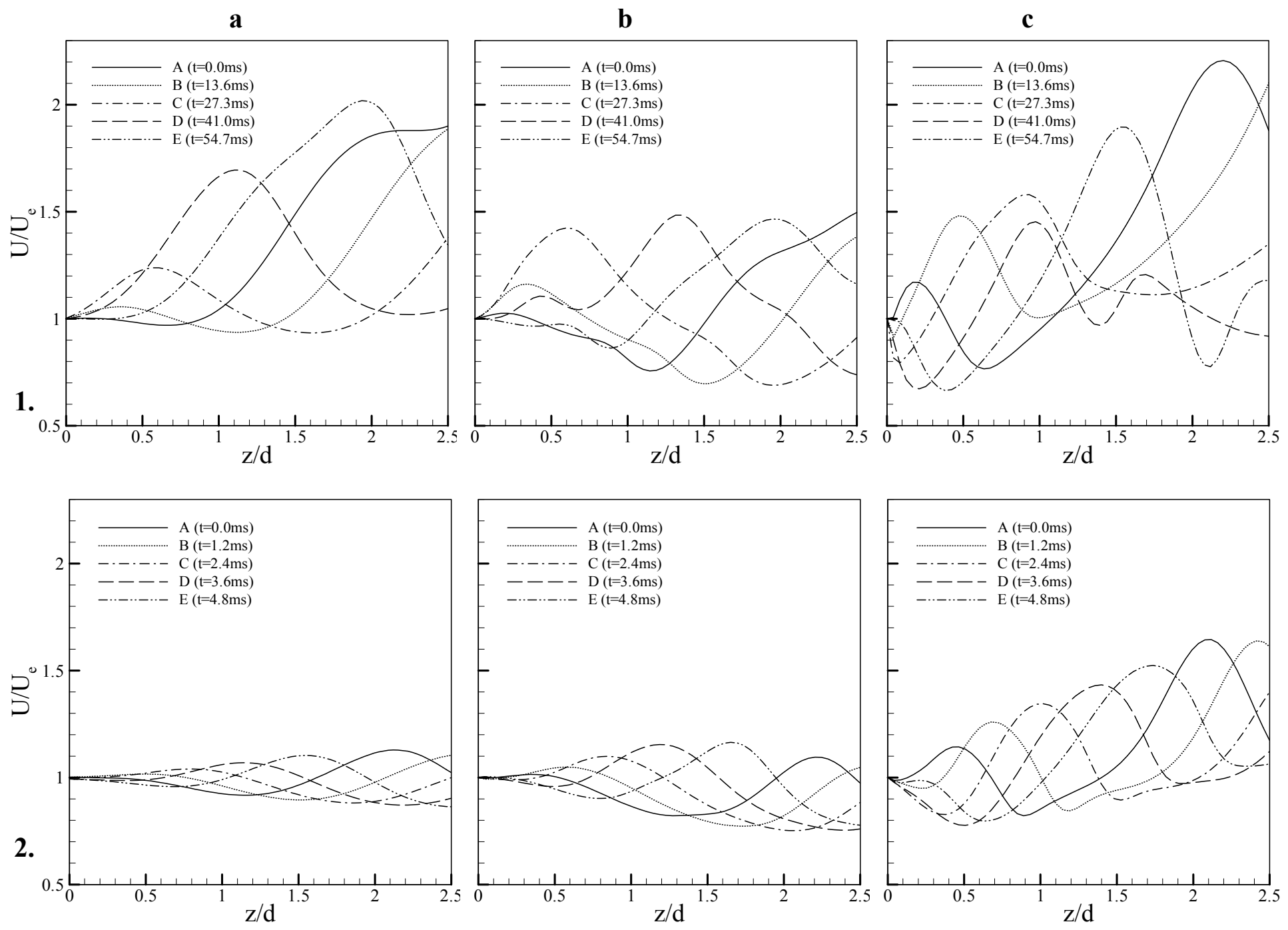

Fig.10, AIAA 
a.
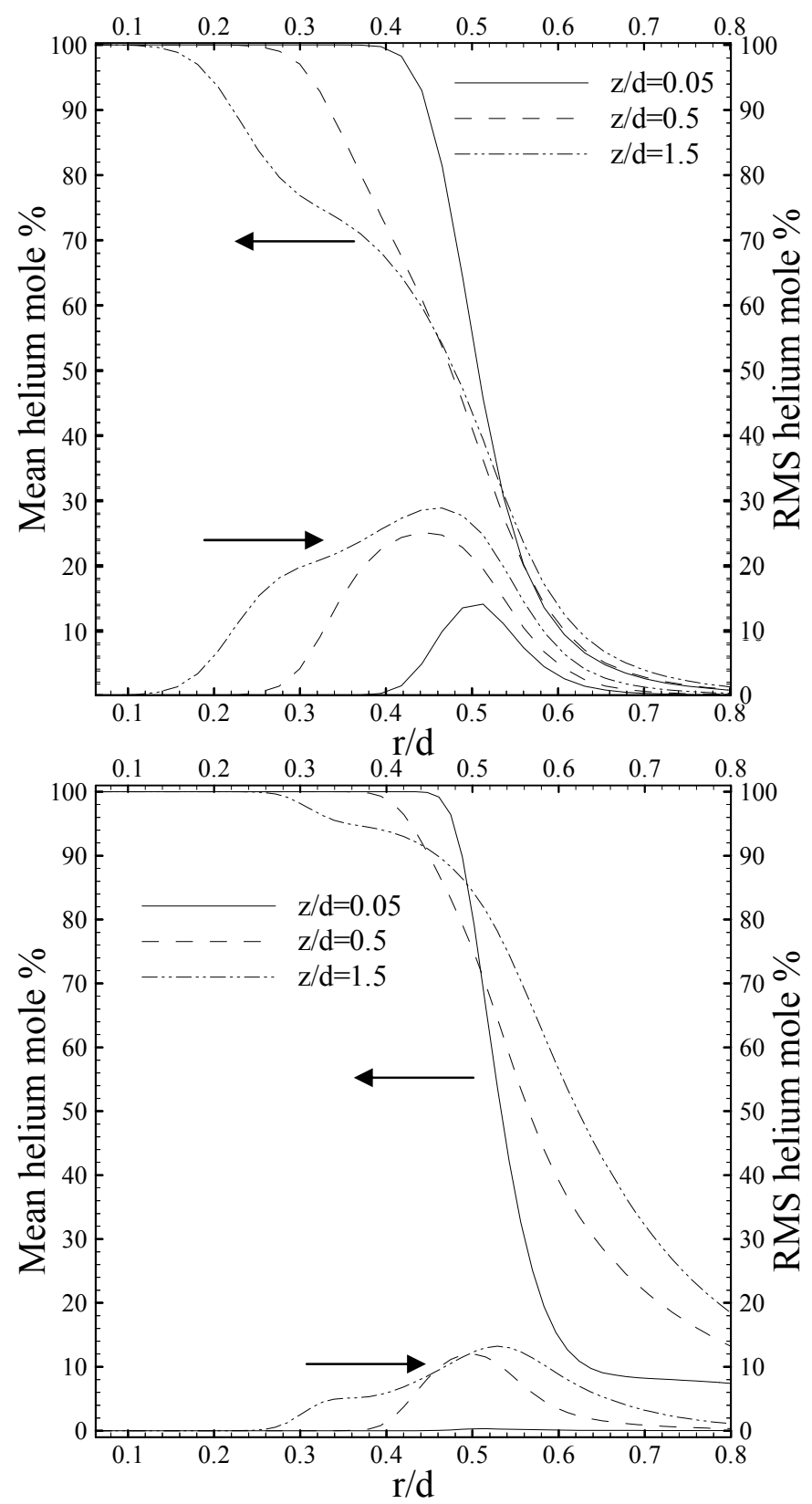
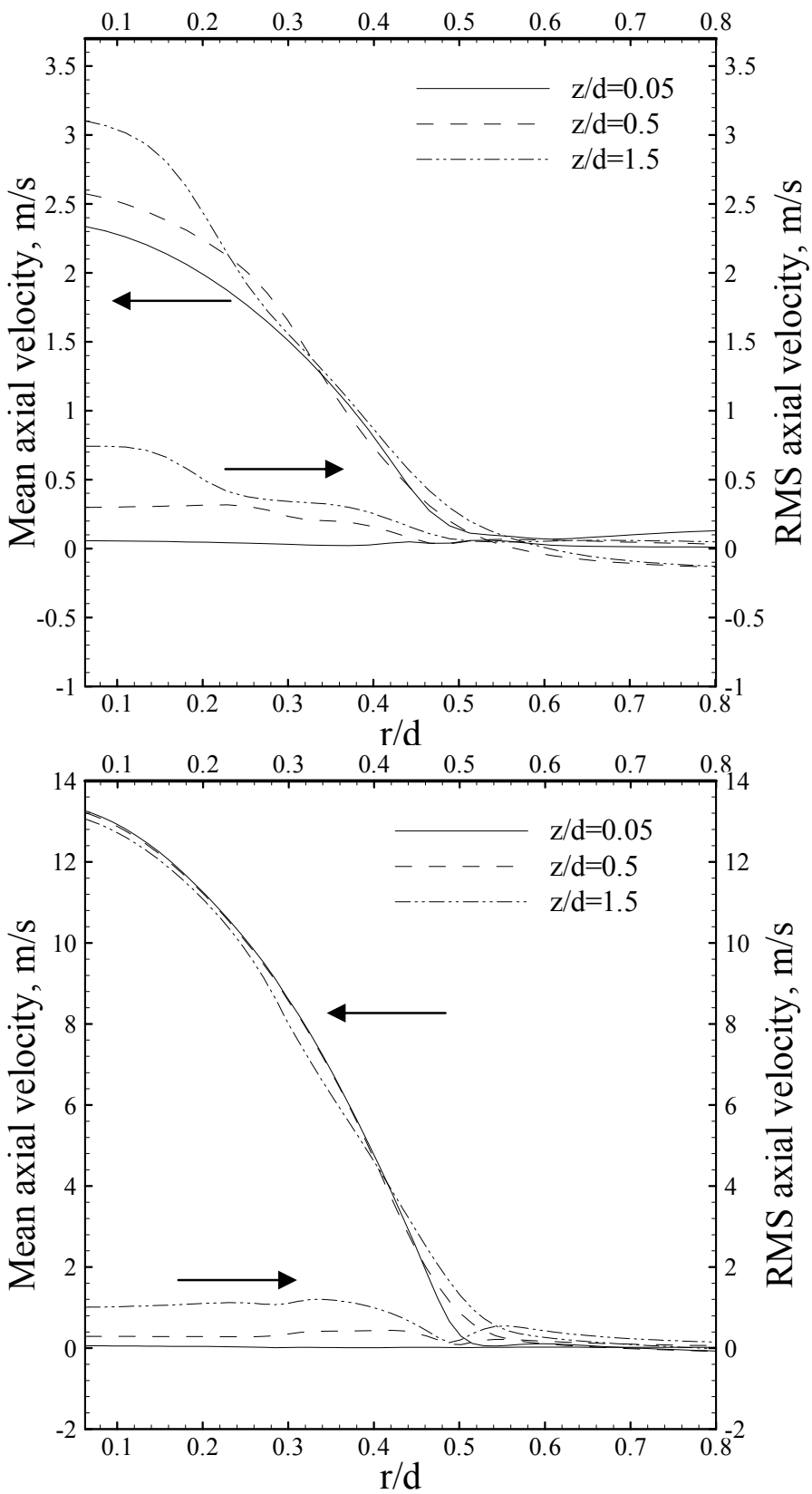

Fig.11, AIAA 

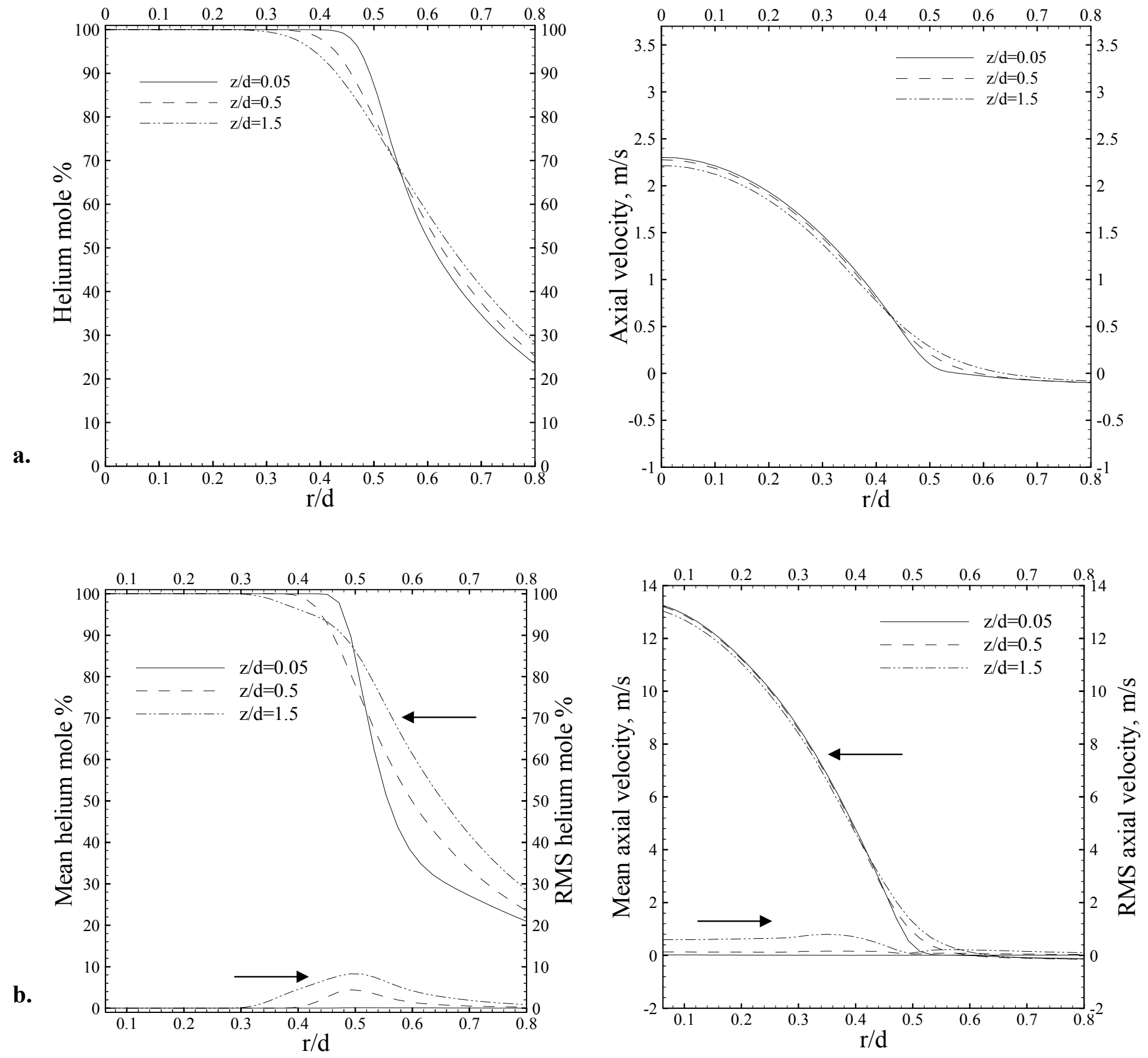

Fig. 12, AIAA 